\title{
Combined size and texture-dependent deformation and strengthening mechanisms in $\mathrm{Zr} / \mathrm{Nb}$ nano-multilayers
}

\author{
M. Callisti ${ }^{1, *}$, T. Polcar ${ }^{1,2}$ \\ ${ }^{1}$ Engineering Science, Faculty of Engineering and the Environment, University of Southampton, \\ Southampton SO17 1BJ, UK \\ ${ }^{2}$ Department of Control Engineering, Faculty of Electrical Engineering, Czech Technical \\ University in Prague, Technická 2, Prague 6, Czech Republic
}

\author{
* Corresponding Author Information \\ Dr. Mauro Callisti \\ M.Callisti@soton.ac.uk \\ Faculty of Engineering and the Environment, University of Southampton, Highfield \\ Southampton SO17 1BJ, UK \\ Building 7 / Room 4027 - Mailpoint M7, Southampton, Hampshire SO171BJ \\ United Kingdom \\ Phone: +44 (0)23 80594438
}

\begin{abstract}
A combination of transmission electron microscopy analyses and nanomechanical measurements was performed in this study to reveal deformation and strengthening mechanisms occurring in sputtered $\mathrm{Zr} / \mathrm{Nb}$ nanoscale metallic multilayers (NMMs) with a periodicity $(L)$ in the range $6-167 \mathrm{~nm}$. Electron diffraction analyses revealed a change in the crystallographic orientation of $\alpha$ - $\mathrm{Zr}$ when $L \leq 27 \mathrm{~nm}$, while $\mathrm{Nb}$ structure retained the same orientations regardless of $L$. For $L>60 \mathrm{~nm}$, the strengthening mechanism is well described by the Hall-Petch model, while for $27<L<60 \mathrm{~nm}$ the refined CLS model comes into picture. A decrease in strength is found for $L<27 \mathrm{~nm}$, which could not be simply explained by considering only misfit and Koehler stresses. For $L \leq 27 \mathrm{~nm}$, plastic strain measured across compressed NMMs revealed a change in the plastic behaviour of $\alpha$-Zr, which experienced a hard-to-soft transition. At these length scales, the combination of two structural factors were found to affect the strength. These relate to the formation of weaker interfaces which extend the effective distance between strong barriers against dislocation transmission, thus producing a softening effect.
\end{abstract}


The second effect relates to the crystallographic orientation change exhibited by $\alpha$-Zr for $L<27 \mathrm{~nm}$ with a consequent change of the dominant slip system. The actual strength at these smaller length scales was effectively quantified by taking these structural aspects into account in the interface barrier strength model.

Keywords: Nanostructured metals, Crystallographic orientation, Plastic deformation, Nanoindentation, Transmission Electron Microscopy (TEM).

\section{Introduction}

One of the core objectives in materials science and engineering is to find a correlation between fabrication processes, physical properties and microstructure of materials so as to allow design of materials with highly tailored properties for specific engineering applications. Nanoscale metallic multilayers (NMMs) represent a relatively new class of heterogeneous materials widely studied, often as model materials, to understand the relationship between intrinsic materials properties (grain size, textures, interfaces, etc.) and the corresponding physical properties, with particular emphasis on mechanical strength [1]. Materials with such architecture may have future applications in various fields ranging from electronics and computer science (micro/nano-electromechanical systems [2], magnetic data storage [3, 4]), hydrogen storage [5, 6], tribology [7] and nuclear industry [8-15]. Most of these applications require properties such as high strength, toughness, wear resistance and structural stability in demanding environments. To this aim, structural and mechanical properties of NMMs were widely investigated and correlated. In particular, different models were proposed to describe the strengthening mechanisms as a function of the individual layer thickness $(h)$ [16]. The Hall-Petch (H-P) model was extensively used to describe the strengthening effect originating from dislocations pile-up at grain boundaries (GBs) [17]. This model was also advantageously used for NMMs with $h$ above $\sim 50 \mathrm{~nm}$ [18]. At this length scale, the flow strength changes linearly with $h^{-1 / 2}$ 
and the H-P slope is often used to predict the interface barrier strength and the peak strength of NMMs [19]. However, these predictions were found to deviate significantly from the measured values when NMMs presented specific intrinsic structural features (grain size $<h$, twins, etc.), which further contributed to strengthen the NMMs [19-24]. As $h$ decreases below few tens of nanometres, measured strength deviates from the linear (H-P) model due to the lack of sufficient dislocations to pile-up at interfaces and GBs. A confined layer slip (CLS) model derived from the Orowan bowing of single dislocations was proposed [16]. In this model the change in strength as a function of $h$ is taken into account by assuming that movement of single dislocations is confined within the layer with a hairpinlike path, since the stress required to transmit dislocations across interfaces is higher than that required to bow dislocations in the layers. The CLS model was subsequently refined to take into account interaction between dislocations and stresses originating from interfaces [16]. When $h$ is reduced below $\sim 5 \mathrm{~nm}$, dislocations transmission across interfaces becomes the dominant mechanism responsible for the strength of NMMs [16, 17]. At this small length scale the strength becomes independent of $h$ and typically reaches a plateau for incoherent systems [25-27] or softening for coherent systems due to the formation of transparent (to dislocation transmission) interfaces [27, 28]. The strength of NMMs in this case is affected by different factors including the Koehler stress [2830], misfit dislocations [24, 28], chemical stress [28, 31, 32], coherency stress [28, 33] and twinning $[23,24,34,35,36]$.

There is a larger number of studies focused on the correlation between structure and mechanical properties of cubic systems $(b c c / f c c$ [16, 24, 37, 38], fcc/fcc [23, 39-41] and $b c c / b c c$ [23, 42]). Only recently, cubic/hcp systems started to attract some attention among the scientific community. NMM systems combining bcc/hcp (Mg/Nb [43], Co/Mo [44]), fcc/hcp (Cu/Ta [45], Cu/Zr [46-49]) and hcp/hcp (Mg/Ti [20]) were studied. In the latter case, no strength plateau was found and the strength kept increasing for smaller $h(\sim 2.5 \mathrm{~nm})$, while for larger $h$ the models mentioned above were successfully used to describe the strength vs $h$ relationship. On the other hand, for $\mathrm{Cu} / \mathrm{Zr}$ [36] a 
strength plateau was found, although in this particular case formation of nano-twins in Cu layers influenced the strength of the system and refinements on the CLS model were needed to take the effect of nano-twins into account. Peak hardness of Mg/Nb NMMs as a function of $h$ was found to be well-described by the H-P model (for large $h$ ) and Koehler stress (for small $h$ ) [43], although a metastable structure of $b c c \mathrm{Mg}$ formed at interfaces for $h=5 \mathrm{~nm}$ further strengthened the barrier against dislocation transmission. From this brief survey of the literature on $h c p$-based NMMs, it is seen that no much insight or consistent trend lines on properties are yet available for NMMs involving hcp metals. Therefore, further studies are needed to understand the role of hcp metals on structure and strength of $h c p$-based NMMs.

This study is aimed at understanding the intrinsic role of $h c p$ structures on structural and mechanical properties of $b c c / h c p$ NMMs at different length scales. Among the possible combinations, $\mathrm{Zr} / \mathrm{Nb}$ NMMs were selected for a number of reasons. The first reason is that the strengthening mechanisms occurring in $\mathrm{Zr} / \mathrm{Nb}$ are not understood. Previous studies on this system focused mostly on structure [50-52], superconductivity [53, 54] and radiation tolerance [9] of $\mathrm{Zr} / \mathrm{Nb}$ NMMs, while no consistent correlation between structure and mechanical properties is reported. The second reason is related to the fact that zirconium and zircalloys (Zr-Nb for instance) are widely employed in nuclear industry due to their small capture cross-section for thermal neutrons, their relatively good high-temperature strength and resistance to corrosion $[55,56]$. In view of the positive role of interfaces against radiation damage (as mentioned before NMMs are largely used as model material to study the role of interfaces on radiation-induced point defects) [10-12, 57-59], Zr-based NMMs could represent a promising candidate material for the future nuclear industry. Therefore, in this study comprehensive structural analyses via analytical scanning transmission electron microscopy (STEM) in combination with nano-mechanical measurements are presented with the aim of correlating very fine structural details with observed mechanical behaviour. Interesting mechanisms counterbalancing the well-known sizedependent strengthening effect are revealed at the smaller length scales. 


\section{Experimental details}

The following sections provide a detailed description of the experimental procedures used in this study comprising synthesis of the layered structures and evaluation of their structural and mechanical properties.

\subsection{Materials synthesis}

Magnetron sputtering was employed to fabricate $\mathrm{Zr} / \mathrm{Nb}$ nanoscale metallic multilayers (NMMs). High-purity Zr and Nb targets were used to deposit NMMs with different individual layer thicknesses (h) onto a single-crystal (111) Si substrate. Prior each deposition, substrates were plasma-etched (argon plasma) for 15 minutes to remove native contamination. The deposition system was equipped with computer-controlled shutters for each power source, therefore the layered structure was achieved by alternate opening/closing of each shutter. The individual layer thickness was controlled by adjusting the shutter opening time according to the deposition rates measured for the sputtered elements. With a power of $150 \mathrm{~W}$ (d.c.) and at a pressure of $0.5 \mathrm{~Pa}$, Zr and $\mathrm{Nb}$ exhibited a deposition rate of $0.1 \mathrm{~nm} \mathrm{~s}^{-1}$ and $0.15 \mathrm{~nm} \mathrm{~s}^{-1}$, respectively. Before each deposition, the chamber was evacuated to a base pressure of $1 \times 10^{-5} \mathrm{~Pa}$. Depositions were performed in Ar atmosphere without any deliberate heating/cooling of the substrate. The substrate was rotated at $10 \mathrm{rpm}$ to achieve constant layer thicknesses across the substrate. The number of bilayers (Nb on top) was determined to produce a total film thickness in the range $1.35-1.5 \mu \mathrm{m}$.

\subsection{Structural and chemical characterisation}

X-ray diffraction (XRD) analyses were carried out on the as-deposited NMMs by using a Rigaku SmartLab diffraction system (Rigaku Corporation, Japan) with $\mathrm{Cu} \mathrm{K} \alpha$ radiation, while the XRD patterns were analysed by the PDXL software coupled with an ICDD PDF-2 database. 
The diffractometer was operated at a grazing incidence angle $\left(4^{\circ}\right)$ for phase identification as well as with the classical $\theta / 2 \theta$ symmetric configuration to determine the crystallographic texture of the films and possible peaks shift due to in-plane residual stresses. Surface morphology and cross-section of the as-deposited NMMs were observed by using a Zeiss NVision40 Focused Ion Beam (SEM/FIB) system. TEM samples were prepared by FIB (in situ lift-out method) and thinned down to achieve electron transparency by mean of a Ga ion gun operated with a current of $40 \mathrm{pA}$ at $30 \mathrm{kV}$. Transmission electron microscopy (TEM) was used to perform further structural analyses. Preliminary observation and electron diffraction analyses were carried out by using a JEOL JEM3010 at an accelerating voltage of $300 \mathrm{kV}$. Finer observations and elemental analyses were carried out by using a Cs-corrected JEOL ARM200F (cold-FEG) TEM/STEM operated at $200 \mathrm{kV}$ and equipped with a Gatan GIF spectrometer and a $100 \mathrm{~mm}^{2}$ Centurion EDX detector (Thermo Fisher Scientific Inc., Madison, Wisconsin, USA). Phase maps for the as-deposited nano-multilayers were attained by electron energy loss spectroscopy (EELS), where data were acquired with an energy dispersion of $0.1 \mathrm{eV} / \mathrm{channel}$ in the low loss region of the energy spectrum.

\subsection{Mechanical characterisation}

Nanoindentation was employed to measure mechanical properties of the as-deposited films. For each sample, the maximum indentation depth was set in order to avoid substrate effects $(10-15 \%$ of the film thickness) and to indent at least one interface for nano-multilayers with larger periodicities (i.e. $167 \mathrm{~nm}$ ). A loading strain rate (LSR) of $0.1 \mathrm{~s}^{-1}$ was used; the unloading time was kept constant at $5 \mathrm{~s}$

for all cases. At least 10 indents for each load condition were performed. Drift measurements were carried out for $60 \mathrm{~s}$ during the unloading at a load of $10 \%$ of the maximum load. The drift rate was calculated from a linear regression of the displacement vs time data and used to correct nanoindentation data. The Berkovich tip was calibrated before tests by using a standard fused silica 
sample. Data were corrected for frame compliance before calculation of mechanical properties based on the procedure outlined by Oliver and Pharr [60].

Indentation creep tests were carried out with a constant load (depth of $\sim 10-15 \%$ of the film thickness) and a constant LSR of $0.2 \mathrm{~s}^{-1}$. The depth at maximum load was recorded for $100 \mathrm{~s}$. The imposed strain rate $(\dot{\varepsilon})$ and the average stress $(\sigma)$ durind indentation creep were calculated respectively by the following equations [61]:

$\dot{\varepsilon}=\frac{1}{h(t)} \frac{d h(t)}{d t}, \sigma=\frac{P_{\max }}{A_{c}}$

Where $h$ is the instantaneous indenter displacement, $P_{\max }$ is the maximum indentation load kept constant during creep test, $A_{c}$ is the contact area for the specific tip used in this study. In order to calculate the strain rate, displacement vs time curves were fitted by the following empirical law:

$h=h_{0}+x\left(t-t_{0}\right)^{y}+z t$

where $h_{0}, x, y$, and $z$ are fitting constants, while $t_{0}$ is the starting time for creep test. The strain-rate sensitivity $(m)$ was determined from the slope of the double logarithmic plot of hardness $(H)$ and strain rate $(\dot{\varepsilon})$ under isothermal conditions based on Eq. (3) [19], while the activation volume was determined similarly by using Eq. (4) [19].

$m=\frac{\partial \ln (H)}{\partial \ln (\dot{\varepsilon})}$

$V^{*}=\frac{2.7 \sqrt{3} k T}{H} \frac{\partial \ln (\dot{\varepsilon})}{\partial \ln (H)}$

where $k$ is the Boltzmann constant and $T$ is the absolute temperature. In Eq. (4), it is assumed that the yield strength of the film correspond to $H / \alpha$, where $\alpha$ is the Tabor factor. In order to explore the creep mechanism occurring in NMMs with $h$, the creep stress exponent $(n)$ was determined from the displacement vs time curves. The steady-state creep is described by an empirical power-low between $\dot{\varepsilon}$ and $\sigma$ as follows [19].

$\dot{\varepsilon}=A \sigma^{n}$ 
where $A$ is a material-related constant. In this case, stress was calculated as a function of time during the hold period for a constant load $\left(P_{\max }\right)$ by using Eq. (1) and divided by a Tabor factor of 2.7. The creep stress exponent was determined from the slope of the $\ln (\dot{\varepsilon})$ vs $\ln (H / 2.7)$ curves. An example of the procedures mentioned above is presented in Fig. S1.

\section{Results}

\subsection{As-deposited structures}

Fig. 1 illustrates the XRD patterns $\left(\theta / 2 \theta\right.$ data) in the range $2 \theta=30-45^{\circ}$, where it is seen that for $L$ $\geq 27 \mathrm{~nm},(0002)_{\mathrm{Zr}}$ and (110) $)_{\mathrm{Nb}}$ textures are parallel (and normal to the film growth direction) and represent the major Bragg diffraction peaks in the patterns. A similar structure was reported for $\mathrm{Mg} / \mathrm{Nb} \mathrm{NMMs}$ [43]. On the other hand, satellite peaks populated the patterns around the main Bragg peaks for $L=12$ and $6 \mathrm{~nm}$. These features suggest formation of a superlattice structure [63]. XRD patterns for $L \leq 27 \mathrm{~nm}$ show a peak intensity evolution in relation to $L$. In particular, for $L=12 \mathrm{~nm}$ a clear decrease in intensity for $(0002)_{\mathrm{Zr}}$ and $(110)_{\mathrm{Nb}}$ is observed together with the development of satellite peaks. For $L=6 \mathrm{~nm}$, satellite peaks become even more prominent and the main Bragg peaks disappear. These observations suggest that for smaller $L(\leq 12 \mathrm{~nm})$, there is an increased degree of interfacial coherency between the layers. For $L=12 \mathrm{~nm}$, a symmetric distribution of superlattice peaks up to the second order emerged around the $(110)_{\mathrm{Nb}}$ texture. Superlattice peaks were also identified by using the following equation [63]:

$\sin \theta_{ \pm}=\sin \theta_{B} \pm \frac{m \lambda}{2 L}$

Where $\theta_{ \pm}$is the $2 \theta$ position of symmetric satellites around the zero order peak, $\theta_{B}$ is the Bragg angle of the main texture, $m$ is the order of the peak, and $\lambda$ is the wavelength of X-rays. The $2 \theta$ positions of superlattice peaks around the (110) $)_{\mathrm{Nb}}$ texture were accurately calculated from Eq. (6) for $L=12 \mathrm{~nm}$. The asymmetrical satellite peaks distribution around the (0002) $\mathrm{zr}$ texture for $\mathrm{L}=12 \mathrm{~nm}$ is attributed to a combination of factors such as layer thickness variation and inhomogeneous strain distribution 
across interfaces [64], as will be discussed later. On the other hand, for $L=6 \mathrm{~nm}$, peaks associated with the main texture (indicated as order " 0 ” peak in Fig. 1) disappeared without significant changes in the position of satellite peaks and an asymmetric peak in the $2 \theta$ range $\sim 35-37^{\circ}$ emerged. Deconvolution of the measured XRD profile (inset in Fig. 1) shows, beside satellite peaks, the presence of two overlapped peaks in the $2 \theta$ range $\sim 35-37^{\circ}$. These peaks are located at $2 \theta=35.65$ and $36^{\circ}$, which correspond to a $d$-spacing of 0.2516 and $0.2492 \mathrm{~nm}$, respectively. Tab. 1 summarises further information extracted from the patterns shown in Fig. 1. The crystallite size $(c)$ for the constituent elements was calculated by using the Sherrer equation [65]. As expected, $c$ decreased for smaller periodicities. For $L \geq 27 \mathrm{~nm}$ there was no significant variation of $d$-spacing for $\mathrm{Zr}$ and $\mathrm{Nb}$. On the other hand, for $L=12 \mathrm{~nm}(0002)_{\mathrm{Zr}}$ and (110) $)_{\mathrm{Nb}}$ peaks (indicated as “0” order peak in Fig. 1) shifted towards opposite $2 \theta$ angles, as also documented by the variation in the corresponding $d$ spacing (Tab. 1). Based on these observations, both constituent layers were subjected to in-plane compressive stresses for any $L$. However, for decreasing $L$ from 27 to $12 \mathrm{~nm}, \alpha$-Zr lattices experienced an in-plane stretching (parallel to the basal plane and interfaces) while Nb lattices were subjected to an even increased in-plane compressive stress. Formation of coherent interfaces for $\mathrm{L} \leq 12 \mathrm{~nm}$ was accompanied by severe distortions of the lattice structure for $\alpha-\mathrm{Zr}$ and $\mathrm{Nb}$, thus leading to an appreciable and opposite change of the stress state inside the constituent layers.

Because of the similar structure found for $L \geq 27 \mathrm{~nm}$, cases such as $L=27$ and $60 \mathrm{~nm}$ are often used in this study as a reference to be compared to cases such as $L=12$ and $6 \mathrm{~nm}$. Selected area diffraction (SAD) pattern for $L=60 \mathrm{~nm}$ (Fig. 2a) exhibits a six-fold symmetry, with the following orientation relationship: $(0002)_{\mathrm{Zr}} / /(110)_{\mathrm{Nb}}$ and $<0001>_{\mathrm{Zr}} / /<110>_{\mathrm{Nb}}$. For $L=6 \mathrm{~nm}$ (Fig. 2b), the same texture as for $L=60 \mathrm{~nm}$ with a six-fold symmetry is found; however, only one broad diffraction ring was observed along the growth direction (I in Fig. 2b), which reflects the coherent structure formed between constituent elements. A line profile across the ring (II in Fig. 2b) shows overlapping of two diffraction rings, thus supporting the deconvolution reported in Fig. 1. Cross-sectional HAADF- 
STEM images of some layered structures are shown in Fig. 3. Because of the higher density, Nb appears brighter in HAADF contrast. A less pronounced columnar structure and waviness formed for larger $L$. High resolution SEM images (not reported) of the top surfaces revealed larger columnar grains for larger $L(\sim 60 \mathrm{~nm}$ for $L=60 \mathrm{~nm})$, while for $L=6 \mathrm{~nm}$ many columnar grains with a diameter ranging between $10-30 \mathrm{~nm}$ were observed. By comparing Fig. $3 \mathrm{a}-\mathrm{d}$, it is evident that the competitive columnar growth normal to the substrate intensified for smaller $L$.

$\mathrm{Zr}-\mathrm{Nb}$ is an immiscible system characterised by a positive heat of formation (6 $\mathrm{kJ} \mathrm{mol}^{-1}$ ) [66]. However, non-equilibrium materials fabrication techniques such as magnetron sputtering allows producing solid solutions even between immiscible elements. In this study, no evidence of mixed boundary layers is found and chemically sharp interfaces are produced even for smaller periodicities (see insets in Fig 3a and 3c). Finer details on the nanostructure and atomic arrangements between layers are reported in Fig. 4. For $L=6 \mathrm{~nm}$, the waviness is found to cause a significant curvature of the layers (Fig. 4a). In most cases Nb structure is imaged along the [111] zone axis with (110) plane parallel to the substrate, although cubic structure oriented along the [001] zone axis is also imaged. For $L \geq 27 \mathrm{~nm}$, XRD analyses (Fig. 1) clearly indicated the prevalence of (0002) $\mathrm{zr}$ and (110) $)_{\mathrm{Nb}}$ textures, which degenerated in one asymmetric peak for $L=6 \mathrm{~nm}$. Fig. $4 \mathrm{~b}$ shows the nanostructure formed for $L=27 \mathrm{~nm}$ with Zr layers grown with the $c$-axis (hcp structure) normal to the $\mathrm{Nb}$ (layer) substrate, while Nb exhibited the (110) texture. It corroborates the orientation relationship identified previously by XRD analyses (i.e. (0002)hcp//(110)bbc). GBs in the constituent layers did not line up each other (Fig. 4a), especially for $L=27 \mathrm{~nm}$, and the lateral grain size (size parallel to the interface) for $\mathrm{Nb}$ layers was often found to be comparable to $L$. On the other hand, $\mathrm{Zr}$ layers exhibited a lateral grain size comparable to the $\mathrm{Zr}$ layer thickness $\left(h_{Z r}\right)$. 


\subsection{Structural evolution at small length scales}

XRD and S/TEM analyses indicated that structural changes occurred when $L$ is decreased below 27 nm. Lowe and Geballe [53] showed experimentally that when $L$ was reduced below $~ 3.1 \mathrm{~nm}$ (in a nano-multilayer with the same $h$ between constituent layers), the $\mathrm{Nb} / \mathrm{Zr}$ (bbc/hcp) structure transformed into a metastable $\mathrm{Nb} / \mathrm{Zr}(b c c / b c c)$ structure, where the $\mathrm{Zr}$ phase transition seemed to be driven by diffusion of $\mathrm{Nb}$ in $\mathrm{Zr}$ layers. On the other hand, Thompson et al. [50] combined a thermodynamic approach with the linear elasticity theory to study the stability of $b c c \mathrm{Zr}$ in $\mathrm{Zr} / \mathrm{Nb}$ NMMs by varying the Nb volume fraction. Based on the proposed biphase diagram (bcc Nb/hcp $\mathrm{Zr}$ and $b c c \mathrm{Nb} / b c c \mathrm{Zr}$ ), for $\mathrm{Nb}$ volume fractions reported here for $\mathrm{Zr} / \mathrm{Nb}_{6}$, $\mathrm{Zr} / \mathrm{Nb}_{12}$ and $\mathrm{Zr} / \mathrm{Nb}_{27}$ films listed in Tab. 1, a bilayer (or $L$ ) thickness below $\sim 2.9 \mathrm{~nm}$ would be necessary to have a stable $b c c \mathrm{Zr}$ structure. To further proof the absence of $b b c \mathrm{Zr}$ in NMMs studied here, we consider the possibility of $\mathrm{Zr}$ to growth epitaxially over $\mathrm{Nb}$ for small $h$. We use the following equation to estimate the critical thickness $h_{c r}$ below which epitaxial growth takes place [20]:

$h_{c r}=\frac{b}{2 \pi \xi} \ln \left(\frac{\sqrt{2} h_{c r}}{b}\right)$

where $b$ is the length of the Burgers vector and $\xi$ is the misfit strain between constituents. For $b=$ $0.3232 \mathrm{~nm}$ and $\xi=9 \%$ a critical thickness of $\sim 0.6 \mathrm{~nm}$ is estimated. This value is far below the smallest layer thickness (3 nm) considered in this study. Therefore, misfit dislocations at $\mathrm{Zr} / \mathrm{Nb}$ interfaces are expected to form and release the interfacial stress due to lattice misfit between constituent layers. As a consequence, a stress-assisted phase transition (hcp-to-bcc) in Zr layers is ruled out for any of the cases under study. In order to shed light on the structural evolution observed in Fig. 1 for small $L$, a more detailed crystallographic analysis was performed on NMMs with $L=27$ and $6 \mathrm{~nm}$. Fig. 5a shows the layered structure formed for $L=27 \mathrm{~nm}$, where $(0002)$ zr planes are oriented parallel to the substrate (c-axis of the $\alpha$-Zr structure about normal to the substrate). While

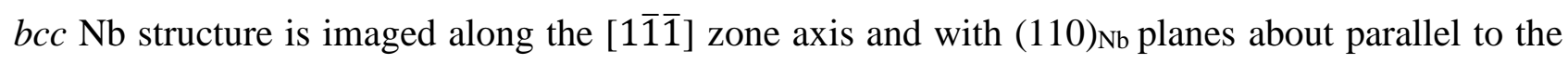
substrate. The FFT in Fig. 5b corroborates the XRD analyses reported in Fig. 1. On the other hand, 
Fig. 5c shows the atomic arrangements typically observed for $L=6 \mathrm{~nm}$. Here, Nb layers are imaged along two zone axes (i.e. [1 $\overline{1} \overline{1}]$ for $\mathrm{Nb}$ located on the top, and [001] for $\mathrm{Nb}$ located on the bottom). Fig. 5d shows the crystallographic orientations observed for $L=6 \mathrm{~nm}$, where the FFT consists of three superimposed patterns. The Zr layer exhibits a $c$-axis nearly parallel to the substrate and an orientation relationship with $\mathrm{Nb}$ (top) as follows: $(10 \overline{1} 0)_{\mathrm{Zr}} / /(110)_{\mathrm{Nb}}$ and $<01 \overline{1} 1>_{\mathrm{Zr}} / /<0 \overline{1} \overline{1}>_{\mathrm{Nb}}$. This analysis suggests that while $\mathrm{Nb}$ structure does not experience appreciable orientation changes in relation to $L, \alpha$-Zr structure undergoes a substantial crystal rotation for $L=6 \mathrm{~nm}$ (i.e. $c$-axis of the $h c p$ structure away from the normal to the substrate). As predicted from the value estimated for $h_{c r}$, misfit dislocations form at interfaces. However, because of the two major orientations exhibited by bcc Nb structure (Fig. 5d), different dislocation densities are expected along interfaces. The distance between adjacent misfit dislocations can be estimated by the following equation [20]:

$s=\frac{b}{\xi}$

In Fig. 5c the two frequently observed interfaces are indicated with I.1 and I.2. Based on HR-STEM observations, the average distances between misfit dislocations along interfaces are $s_{I .1} \approx 2 \mathrm{~nm}$ and $s_{I .2} \approx 4 \mathrm{~nm}$, therefore different misfit strains are expected on these interfaces. These structural features (i.e. crystal rotation for $\alpha-\mathrm{Zr}$ and different dislocation densities at interfaces) affect dislocations movements inside the layers and across interfaces and therefore the deformation mechanisms and strength of the layered structures, as will be discussed in the following sections.

\subsection{Mechanical properties}

\subsubsection{Hardness and elastic modulus}

Mechanical properties measured by nanoindentation are listed in Table 2. The hardness is found to increase with decreasing $L$, thus corroborating the commonly observed size-dependent hardening effect in NMMs [16]. From the lowest value (4.2 $\pm 0.1 \mathrm{GPa})$ for $\mathrm{L}=167 \mathrm{~nm}$ a peak hardness of 5.2 $\pm 0.2 \mathrm{GPa}$ is reached for $L=27 \mathrm{~nm}$, while with a further decrease of $L$ a drop in hardness occurred. 
The peak hardness measured for $\mathrm{Zr} / \mathrm{Nb}$ is lower compared to that reported for $\mathrm{Cu} / \mathrm{Zr}$ (with $L=10 \mathrm{~nm}$ ) [67], but higher than those reported for $\mathrm{Mg} / \mathrm{Nb}$ (with $L=10 \mathrm{~nm}$ ) [43] and $\mathrm{Mg} / \mathrm{Ti}$ (with $L=5 \mathrm{~nm}$ ) [20]. The elastic modulus $E$ of the layered structures exhibited a similar trend as the hardness (Tab. 2). Values of $E$ reported in Tab. 2 are very close to those calculated by using the rule-of-mixture (119 GPa for $L=6 \mathrm{~nm}$ ) by assuming a modulus of 125.1 [68] and $113 \mathrm{GPa}$ [69] for Zr and Nb, respectively. The rule-of-mixture was also found in other studies [20] to estimate well the modulus of layered structures.

In Fig. 6a, the flow strength $(\sigma=H / \alpha)$ computed by using a Tabor factor $(\alpha)$ of 2.7 is reported against

$h_{N b}^{-1 / 2}$ for $\mathrm{Zr} / \mathrm{Nb}$ NMMs. A straight line can be drawn for $0.17 \leq h_{N b}^{-1 / 2}$ (corresponding to $h_{N b} \geq 24$ $\mathrm{nm})$, thus reflecting the H-P relationship $\left(\sigma \sim h^{-1 / 2}\right)$. The resulting H-P slope $\left(k_{\sigma}\right)$ is $\sim 1.35 \mathrm{GPa}$ $\mathrm{nm}^{1 / 2}$, which is appreciably lower compared to values reported in other studies for bcc/hcp NMMs [20,43]. With a further decrease of $h$ the hardness and strength of the layered structure continued to increase, but deviating from the linear H-P model. In fact, the model underestimates the flow strength when $h_{N b}=11 \mathrm{~nm}\left(h_{Z r}=16 \mathrm{~nm}\right)$. It suggests that the deformation mechanism differs from the classical dislocations pile-up at grain boundaries when $L$ is decreased below $60 \mathrm{~nm}\left(h_{Z r}=36 \mathrm{~nm}\right.$ and $h_{N b}=24$ $\mathrm{nm})$. A maximum flow strength of $1.9 \mathrm{GPa}$ is reached for $L=27 \mathrm{~nm}$. The strengthening mechanisms in relation to the length scale are discussed in section 4.

\subsubsection{Indentation creep}

Depth vs time data were treated by using the procedure described in section 2.3 to determine material properties such as strain-rate sensitivity $m$, activation volume $V^{*}$ and creep stress exponent $n$. However, it was recently demonstrated that the evaluation of $n$ by using indentation creep data leads to a significant overestimation of the stress exponent [70]. Therefore, this parameter is only used here for a qualitative comparison between NMMs with different $L$. We found that with decreasing $L$, the resistance to creep ( $n$ ) gradually increased to reach a peak for the nano-multilayer with $L=27 \mathrm{~nm}$ 
(the hardest film). A further decrease of $L$ led to a significant drop of $n$ below the values found for all other cases. Similar trends were also reported for other nano-multilayers combining $f c c / h c p$ [71] and $f c c / f c c$ [72] structures. Such tendency matches well with that exhibited by the hardness (Fig. 6a), i.e. the harder the more creep resistant. Average activation volume $\left(V^{*}\right)$ and strain-rate sensitivity $(m)$ are listed in Tab. 2. Calculated values are also plotted in relation to the most sensible parameter, i.e. $h_{N b} / L$. Fig. 6b shows an increasing (decreasing) tendency for $m\left(V^{*}\right)$ with $h_{N b} / L$ as also observed for $\mathrm{Cu} / \mathrm{Zr}$ NMMs in relation to $h_{C u} / L$ [48]. The activation volume decreases from $\sim 9.4 b^{3}$ to $\sim 3 b^{3}$ for larger $h_{N b} / L$, as also reported for $\mathrm{Cu} / \mathrm{Zr}$ NMMs for similar length scales [73], although an exception is found for the film with $h_{N b}=11 \mathrm{~nm}$ (or $L=27 \mathrm{~nm}$ ). It indicates that in this particular case, the relative amount of $\mathrm{Nb}$ is not the only factor affecting $V^{*}$. It was suggested [74] that when $V^{*}$ exceeds $\sim 100 b^{3}$ the dominant deformation mechanism is attributable to the intersection of forest dislocations generated inside the grains. On the other hand, when $V^{*}$ diminishes below $1 b^{3}$, GB sliding and diffusion (Coble creep) are the dominant mechanisms [74]. In the intermediate range $1-100 b^{3}$, the deformation mechanism tends to be driven by cross-slip of screw dislocations $\left(\mathrm{V}^{*} \sim 10-100 b^{3}\right)$, or dislocation emission from interfaces and GBs $\left(\mathrm{V}^{*} \sim 1-10 b^{3}\right)[75,76]$. According to this scenario and to the values of $V^{*}$ listed in Tab. 2 for $\mathrm{Zr} / \mathrm{Nb} \mathrm{NMMs}$, dislocation emission from GBs and interfaces should be expected for any intrinsic length scale except that for $h_{N b}=11 \mathrm{~nm}(L=27 \mathrm{~nm})$, where a mixed mechanism (dislocations emission from GBs/interfaces and screw dislocations generated inside the grains) can occur. This mechanism could be the physical reason behind the measured peak hardness (Fig. 6a) and high $\mathrm{V}^{*}$ value for $\mathrm{L}=27 \mathrm{~nm}$ (Fig 6b). The hardening effect is caused by the interaction and mutual obstruction between dislocations emitted from GBs/interfaces and existing/developing dislocations inside the grains during nanoindentation. The dependence exhibited by $m$ on $h_{N b} / L$ (Fig. 6b) indicates that even $m$ does not depend only on thermally and/or stress activated processes but also on the relative content of the specific constituent element (i.e. Nb). NMMs with smaller $L$ presented a higher $m$, as also observed for Cu/Zr NMMs [36]. The strain rate sensitivity 
was also plotted against the number of crossed interfaces calculated according to the maximum indentation depth (not shown) and it revealed a sensible increase of $m$ only for $L \leq 12 \mathrm{~nm}$. These observations lead to the conclusion that both relative amount of $\mathrm{Nb}$ and interface density distribution have major effects on $m$.

\section{Discussion}

In this section, we present a critical evaluation of presented results by combining experimental evidence with models. Particular emphasis is given to the relationship between structural evolution of the layered structures in relation to $h$ and the corresponding strengthening and deformation mechanisms activated during nanoindentation.

\subsection{Strengthening mechanisms}

The layered structure adds extra strength with respect to that of single constituent elements. The ruleof-mixture (ROM) is often used to estimate the strength of nanocomposites:

$\sigma_{R O M}=V_{Z r} \sigma_{Z r}^{y}+V_{N b} \sigma_{N b}^{y}$

where $V$ is the volume fraction and $\sigma^{y}$ is the yield strength of the constituents. By using the yield strength of constrained $\mathrm{Zr}$ and Nb monolithic layers of 1.6 GPa and 1.25 GPa, respectively [47], for $L=167 \mathrm{~nm}\left(V_{Z r} / V_{N b} \approx 1.46\right)$ the strength estimated by the ROM (1.46 GPa) matches reasonably well the experimental value (1.52 GPa). On the other hand, the strength estimated by the same equation deviates from the measured strength when applied to NMMs with smaller L. Specifically, strengths of $\sim 1.4 \mathrm{GPa}$ and $\sim 1.46 \mathrm{GPa}$ are calculated for $L=6 \mathrm{~nm}\left(V_{Z r} / V_{N b}=1\right)$ and $L=27 \mathrm{~nm}$ $\left(V_{Z r} / V_{N b} \approx 1.45\right)$, respectively. In both cases the ROM underestimates the strength of approximately 23\%. These simple calculations lead to the conclusion that different strengthening mechanisms take place on different length scales. For NMMs, the nature of interfaces is well-known to play an important role. In particular, coherent (or transparent) interfaces facilitate dislocation transmission 
from one layer to the other when compared to semi-coherent and incoherent interfaces (opaque to dislocations) [16]. In Fig. 7 some minor variations in strength for $L \leq 27 \mathrm{~nm}$ are observed. Zhang et al. [47] proposed another possible mechanism to describe the length-independent strength of multilayers composed of softer/ductile layers and harder/brittle layers for small $h$. This mechanism is known as load-bearing effect, where co-deformation of both constituent layers occurs instead of a preferential deformation. Following this model, $\mathrm{Zr}$ and $\mathrm{Nb}$ layers could be seen as two layers with similar mechanical properties, therefore the resulting strength might be expressed again by the ROM. However, as shown previously, the ROM underestimates the strength at smaller $L$. Therefore, the deformation mechanisms in $\mathrm{Zr} / \mathrm{Nb}$ NMMs at small length scales must be affected by other structural factors.

For $60 \mathrm{~nm} \leq L \leq 167 \mathrm{~nm}$, the H-P model fits well the experimental data (Fig. 6a) with a resulting HP slope $\left(\mathrm{k}_{\sigma}\right)$ of $\sim 1.35 \mathrm{GPa} \mathrm{nm}^{1 / 2}$. This low value compared to many other NMM systems $[20,43]$ is attributed to the large lateral grain size exhibited by Nb layers (Fig. 4), where nucleated dislocations can spread laterally (parallel to interfaces) within the Nb layers till dislocations encounter GBs within the layer. A similar effect was also observed in $\mathrm{Cu} / \mathrm{Ni}$ NMMs subjected to tensile stress [76]. Since the H-P slope represents the strength of GBs against slip transmission, it relates to GB or interface strength by the following equation [16]:

$k=\left(\frac{\tau^{*} \mu b}{\pi(1-v)}\right)^{1 / 2}$

where $\tau^{*}$ is the critical resolved shear stress for slip transmission of dislocations across interfaces, $v$ is the Poisson ratio, $\mu$ is the shear modulus and $b$ is the length of the Burgers vector. To calculate $\tau^{*}$ from Eq. (10), we transform the parameter $k_{\sigma}$ measured experimentally in $k$ for shear stress by using a Taylor factor of 3.1. Calculations of the shear strength are performed by using $\mu_{z r}=33 \mathrm{GPa}, v_{z r}=$ 0.34 and $b_{Z r}=0.3232 \mathrm{~nm}$ (or $\mu_{N b}=37.5 \mathrm{GPa}, v_{N b}=0.4$ and $b_{N b}=0.2868 \mathrm{~nm}$ ) for dislocation pileup assumed to occur in $\mathrm{Zr}$ (or in $\mathrm{Nb}$ ) layers. The resolved shear stress $\tau^{*}$ calculated by assuming dislocation pile-up in any of the constituent layers leads to a large underestimation of the peak strength 
compared to values measured experimentally. Therefore, $k_{\sigma}$ cannot be used to estimate the peak strength for this NMM system. The reason behind the observed deviation is attributable to the lack of a sufficient amount of dislocations piling-up against interfaces when the individual layer thickness is somewhat lower than 50 - $30 \mathrm{~nm}$. A confined layer slip (CLS) model was proposed [16] to describe the dislocation activity and, in particular, to quantify the stress required to propagate a glide loop of Burgers vector confined within one layer. The following equation is used to calculate the normal yield stress $\sigma_{c l s}$ :

$$
\sigma_{c l s}=M \frac{\mu^{*} b}{8 \pi h^{\prime}}\left(\frac{4-v}{1-v}\right)\left[\ln \frac{\alpha h^{\prime}}{b}\right]
$$

where $M$ is the Taylor factor, $\mu^{*}$ is the effective shear modulus of the $\mathrm{Zr} / \mathrm{Nb}$ nano-multilayer calculated by combining shear moduli and volume fractions of the constituent elements as $\left(\mu_{Z r}\right.$. $\left.\mu_{N b}\right) /\left(V_{Z r} \cdot \mu_{Z r}+V_{N b} \cdot \mu_{N b}\right), b$ is the Burgers vector, $h^{\prime}$ is the layer thickness parallel to the glide plane ( $h^{\prime}=h \cdot \sin \varphi, \varphi$ is the angle between the slip plane and the interface) and $v$ is the Poisson ratio. By substituting $M=3.1, \mu^{*}=35, v=0.4, b=0.2868 \mathrm{~nm}, \alpha=0.6$ and $\varphi=60^{\circ}$ in Eq. 11 the corresponding $\sigma_{c l s}$ is plotted in Fig. 7. It is evident that the CLS model underestimates (overestimates) the strength of the $\mathrm{Zr} / \mathrm{Nb}$ NMMs for $h$ smaller (larger) than $10-15 \mathrm{~nm}$. Misra et al. [16] proposed a refined CLS model in order to take into account two more contributions to the CLS stress. The first contribution $(f / h)$ takes into account the interface stress $(f)$ due to the elastic deformation of the interfacial region. The second contribution $(C / \lambda)$ takes into account of possible dislocation-dislocation interactions during the CLS mechanism between existing or formed dislocations along interfaces. Therefore, Eq. 11 is modified as follows:

$\sigma_{c l s}=M \frac{\mu^{*} b}{8 \pi h^{\prime}}\left(\frac{4-v}{1-v}\right)\left[\ln \frac{\alpha h^{\prime}}{b}\right]-\frac{f}{h}+\frac{C}{s}$

where $C=\mu b /(1-v)$ and $s=b / \varepsilon$. By using $\alpha=0.2$, a typical value for $f$ of $2 \mathrm{~J} / \mathrm{m}^{2}$ and $\lambda=23 \mathrm{~nm}$, the result obtained with the refined CLS model is presented in Fig. 7. A much better estimation of the strength is achieved with the refined model for $10 \leq h \leq 30 \mathrm{~nm}$. However, with a further reduction 
of $h$ below $10 \mathrm{~nm}$, the strength estimated by the refined CLS model keeps rising thus deviating from the experimentally measured strength. When $h$ is lowered down to a few nanometres, the strength is predominantly controlled by interfaces and such mechanism is not captured by models described above [16]. The strength of the barrier against single dislocation slip transmission is strongly influenced by the lattice mismatch and shear modulus mismatch between the constituent elements. Therefore, by assuming that the interfacial structure does not change with $h$, the interface barrier strength $\sigma_{I B S}$ can be estimated by taking into account two contributions. The first contribution, known as image or Koehler stress, comes into action when the dislocation moves from the soft to the hard layer, where a repulsive force arises due to the difference in shear moduli between layers. The image stress is quantified by using the following equation [20]:

$\tau_{\text {image }}=\frac{\mu_{N b}-\mu_{Z r}}{\mu_{N b}+\mu_{Z r}} \frac{\mu_{Z r} \sin \varphi}{8 \pi}$

Eq. 13 gives $\tau_{\text {image }}$ of $\sim 0.075 \mathrm{GPa}$; as expected, it is rather small in view of the small shear modulus mismatch between constituent layers. The second contribution arises from the lattice parameters mismatch between constituent layers and therefore from the array of misfit dislocations along interfaces, which can limit the slip transmission of dislocations. The misfit stress is quantified by using the following equation [20]:

$\tau_{\text {misfit }}=\alpha \mu^{*}\left(\xi-\frac{b}{2 h}\right)$

where $\alpha$ is the Saada's constant $(\sim 0.5)$ and $\xi$ is the misfit strain. The interface boundary strength (IBS) stress is calculated then as $\sigma_{I B S}=M\left(\tau_{\text {image }}+\tau_{\text {misft }}\right)$. Accurate evaluation of the misfit strain is not obvious for the particular case under study. In order to estimate $\tau_{\text {misfit }}$ from Eq. 14, the misfit strain is evaluated from Eq. 8, where the average distances between misfit dislocations are measured from HR-STEM images for frequently observed interfaces $\left(s_{I .1} \approx 2 \mathrm{~nm}\right.$ and $\left.s_{I .2} \approx 4 \mathrm{~nm}\right)$. For $\xi_{I .2}^{Z r}=$ $b_{Z r} / s_{I .2} \approx 0.072$ and $\xi_{I .2}^{N b}=b_{N b} / s_{I .2} \approx 0.081$ a misfit stress of $\sim 0.47 \mathrm{GPa}$ and $\sim 0.42 \mathrm{GPa}$ is estimated, respectively. By using the misfit strains calculated above and a Taylor factor of 3.1, a $\sigma_{I B S}$ 
of $1.5\left(\sigma_{I B S}^{\xi_{I .2}^{Z r}}\right)$ and $1.7\left(\sigma_{I B S}^{\xi_{I .2}^{N b}}\right)$ GPa are computed, which are not very far from the measured strength (1.85 GPa) for $L=6 \mathrm{~nm}$. On the other hand, when the misfit strain is calculated by using $s_{I .1}, \sigma_{I B S}$ is largely overestimated ( $5 \mathrm{GPa}$ ) compared to the experimental value. This discrepancy clearly indicates that one of the interfaces (i.e. I.2 in Fig. 5c) is a weaker barrier against dislocation transmission. It extends the effective distance between strong barriers for dislocation slip, thus producing a softening effect. By considering $1.5 \mathrm{GPa}\left(\sigma_{I B S}^{\xi_{I .2}^{Z r}}\right)$ as a more realistic $\sigma_{I B S}$ value originating from the weaker interface (i.e. I.2), the strength calculated by IBS model underestimates experimental values for $L=6 \mathrm{~nm}$. Such discrepancy is attributed to the fact that the model does not take into account the texture evolution experienced by $\alpha$-Zr (Fig. 5), which is also expected to affect the deformation mechanism of $\mathrm{Zr} / \mathrm{Nb}$ NMMs. It is worth mentioning again that interface and GB sliding for $L=6 \mathrm{~nm}$ are ruled out (see section 3.3.2). In order to have a more comprehensive understanding of the deformation mechanisms in $\mathrm{Zr} / \mathrm{Nb}$ NMMs, further structural analyses on compressed NMMs were carried out and presented in the next section.

\subsection{Deformed structures}

In the previous section possible explanations behind the observed decrease in strength for $L<27 \mathrm{~nm}$ were proposed. However, further analyses were needed in order to correlate measured properties with the observed microstructure. To this aim, NMMs with $L=27$ and $6 \mathrm{~nm}$ were plastically deformed by using nanoindentation and afterwards subjected to FIB/STEM analyses. After indentation to a large depth $(1.9 \mu \mathrm{m})$, a pile-up with three external and one internal shear bands (SBs) formed for $L=27$ nm (Fig. 8a). External SBs extended almost through the whole film thickness as observed in crosssection (Fig. 8c), where jumps up to $100 \mathrm{~nm}$ across the SBs are observed without significant rupture of the layers. On the other hand, when SBs became closer each other (region close to the substrate in Fig. 8c), some discontinuities in the layered structure formed. A more significant pile-up with four external and internal SBs formed for $L=6 \mathrm{~nm}$ (Fig. 8b) as highlighted in the cross-section shown in 
Fig. 8d. The number of formed SBs were affected by the individual layer thickness, in agreement with other studies $[77,78]$. This behaviour is attributed to the high interfacial coherency achieved in NMMs with sufficiently small $h$, where dislocations transmission across layers are facilitated (i.e. dislocation crossing interfaces), thus assisting SBs formation [77, 78].

Finer structural analyses were carried out on NMMs $(L=27$ and $6 \mathrm{~nm})$ deformed with a load of 50 $\mathrm{mN}$ (maximum depth of $\sim 600 \mathrm{~nm}$ ). The NMM with $L=27 \mathrm{~nm}$ was subjected to a total plastic strain of $25 \%$ and no signs of pile-up were found on the indented surface (Fig. 9a). Fig. 9b shows the crosssection of the deformed structure, where a noticeable layer compression is observed closer to the film surface. The first 10 bilayers below the surface experienced some ruptures as shown more in detail in Fig. 9c. Plastic strain $\left(\varepsilon_{p}\right)$ of deformed NMMs was calculated based on the layer thickness measured directly on STEM images of compressed samples and results are summarised in Fig. 9d. For the first 10 bilayers below the surface, $\varepsilon_{p}$ could not be quantified accurately due to breakage of some layers. It is seen that severe deformation occurred closer to the film surface, while no appreciable plastic strain was calculated for layers close to the substrate. Although a similar trend is observed for the constituent layers, Nb layers were subjected to a more severe strain, thus suggesting that Nb had a higher control of the deformation mechanism.

The NMM with $L=6 \mathrm{~nm}$ was subjected to a total plastic deformation of $17 \%$ along the loading axis. Pile-up was observed around the indent (Fig. 10a) with formation of an external SB. The latter is shown in cross-section in the inset of Fig. 10b, where no rupture of the layered structure is noticed. In this case, $\varepsilon_{p}$ was calculated by measuring the thickness of individual layers for each constituent element in four different regions (Fig. 10c) across the film thickness (Fig. 10b). In particular, plastic strain reported in Fig. 10d was computed based on an average thickness for compressed $\mathrm{Zr}$ and $\mathrm{Nb}$ layers measured in each window (Fig. 10c). The layer thickness variation for the same element in every window was rather small (see small standard deviation in Fig. 10d). Again a more severe deformation was found in the layers close to the film surface. However, in this case Zr layers were 
more compressed compared to Nb layers. Mixture of the constituent elements after compression is ruled out as demonstrated in Fig. S2. By comparing the plastic strain reported in Fig. 9d and 10d, it is seen that the for $L=27 \mathrm{~nm}, \varepsilon_{p}$ for each constituent layer is higher compared to the case of $L=6$ nm. The reason behind this discrepancy is attributed to SBs formation. In particular, SBs accommodate part of the deformation induced by indentation thus liming plastic strain of the layers. On the hand, for $L=27 \mathrm{~nm}$, at an indentation load of $50 \mathrm{mN}$ no SBs formed (Fig. 9a) and the imposed deformation was accommodated only by the layers.

As referred to above, for $L=27 \mathrm{~nm}$, Nb resulted more strained than $\mathrm{Zr}$ layers, for $L=6 \mathrm{~nm}$ the opposite scenario was found. This result is not correlated to the presence/absence of SBs or nature of interfaces, but instead to the change of the crystallographic orientation experienced by $\alpha-\mathrm{Zr}$ for $L<$ $27 \mathrm{~nm}$ (see section 3.2). Fig. 11 shows the layered structures for $L=27$ and $6 \mathrm{~nm}$ underneath the indented surfaces but in regions of the films where no rupture of the layers occurred. For $L=27 \mathrm{~nm}$, $\mathrm{Nb}$ layers experienced a larger compressive $\varepsilon_{p}$ compared to $\mathrm{Zr}$ as shown in detail in Fig. 11a, where it is also shown that the orientation relationship (0002) $\mathrm{Zr} / /(110)_{\mathrm{Nb}}$ was preserved after compression. On the other hand, a different scenario is observed for $L=6 \mathrm{~nm}$ (Fig. 11b), where Zr layers were occasionally observed to exhibit stress-induced twin structures after compression test as further highlighted in the inset of Fig. 11b. Twins formation (not seen for $L=27 \mathrm{~nm}$ ) is an indication of changes in the deformation behaviour of $\alpha-Z r$.

The yield strength of hcp metals is strongly dependent on the combination of active deformation modes (slip and twinning), which in turn depends on the c/a ratio, available deformation modes, critical resolved shear stress (CRSS) for slip, twin activation stress and orientation of the hcp structure with respect to stresses $[55,79]$. Based on this complex combination of factors, mechanisms such as dislocation reactions, slip-twin and twin-twin interactions can occur simultaneously [79]. For $\alpha$-Zr, the most frequently observed slip systems are the first-order prismatic $\{10 \overline{1} 0\}\langle 1 \overline{2} 10\rangle$ and to a minor extent the pyramidal $\{1 \overline{1} 01\}\langle 11 \overline{2} 0\rangle$ and $\{11 \overline{2} 2\}\langle 11 \overline{2} \overline{3}\rangle$ systems [80]. In most of the loading 
conditions, prismatic slip is dominant and the activation of both prismatic and pyramidal slip systems is strongly dependent upon loading direction in highly textured $\mathrm{Zr}$ [80, 81]. External factors such as temperature and strain rate were also found to affect the deformation mechanism in $\alpha$-Zr. However, hcp metals deformed in a quasi-static mode at ambient temperature, as it is the case in this study, are found to yield mainly by slip and work harden through a combination of slip and twinning [82]. Based on these intrinsic (material dependent) and extrinsic (test dependent) aspects, the major factor to determine the strength of $\alpha-\mathrm{Zr}$ in this study is associated with the crystallographic orientation of $\alpha-\mathrm{Zr}$ with respect to stresses. For $L=27 \mathrm{~nm}$, the basal plane of $\alpha-\mathrm{Zr}$ is oriented normal to the loading direction, therefore basal slip is ruled out as a dominant slip system. Zr samples compressed along the $c$-axis were found to exhibit the highest yield stress and work hardening with respect to other orientations [82]. In view of the limited possibility for a prismatic slip system to be activated due to the unfavourable $c$-axis orientation with respect to the loading direction, pyramidal slip systems are preferred for $L=27 \mathrm{~nm}$. On the other hand, for $L=6 \mathrm{~nm}$, the $\alpha$-Zr structure is very favourably oriented for the prismatic slip to be activated (i.e. $\{10 \overline{1} 0\}\langle 1 \overline{2} 10\rangle)$. However, twins-like atomic arrangements were occasionally observed in $\alpha$-Zr after compression tests (Fig. 11b). It was unexpected, as even when the $h c p$ crystal is oriented in a way that the basal slip is excluded, the stress needed to activate twinning is higher than that needed for slip on non-basal planes [80, 83]. Therefore, twins observed in Fig. 11b must have formed due to local stress concentrations at the $\mathrm{Zr} / \mathrm{Nb}$ interfaces (i.e. single dislocations pushing against $\mathrm{Zr} / \mathrm{Nb}$ interface). Prismatic slip being the less demanding mechanism to be activated compared to deformation twinning $[80,83]$ is the mechanism controlling the deformation process and therefore responsible for the observed decrease in strength for $L<27 \mathrm{~nm}$ (Fig. 7a). Gong et al. [84] investigated the strength of different slip systems in single crystal pure $\alpha$-Zr by microcantilever bending tests. Prismatic slip was found to be the most easy slip system with a critical resolved shear stress (CRSS) of $\sim 153$ MPa. For basal and pyramidal slip a CRSS of $\sim 204$ and $\sim$ 
$532 \mathrm{MPa}$ were reported [84], respectively. In our study, the change in strength $\sigma_{I B S}$ originating from the crystallographic rotation experienced by $\alpha-\mathrm{Zr}$ is taken into account as follows:

$\sigma_{I B S}=M\left(\tau_{\text {Misfit }}+\tau_{\text {Image }}+\tau_{S S}\right)$

where $\tau_{S S}$ represents the shear stress needed to activate the most favourable slip system in the relevant crystal structure. Therefore, by considering a prismatic slip system as the main deformation mechanism in $\alpha$-Zr layers $\left(\tau_{S S}=0.15 \mathrm{GPa}\right)$ [84], $\tau_{\text {image }}=0.075 \mathrm{GPa}$ and $\tau_{\text {misft }}=0.42 \mathrm{GPa}$, a $\sigma_{I B S}=$ $2 \mathrm{GPa}$ is calculated, which matches reasonably well the measured strength.

Our study indicates that the strengthening mechanisms occurring in $\mathrm{Zr} / \mathrm{Nb}$ NMMs can be described by established models (H-P and CLS) for $L \geq 27 \mathrm{~nm}$. For smaller L, we observed drop in hardness and strength caused by structural changes, i.e. formation of weaker interfaces and crystallographic orientation changes for $\alpha$-Zr. To match experimental data, we propose a refined IBS model taking into account only the strength of weaker interfaces (i.e. I2 in this case) as well as stress needed to activate the most favourable slip system in $\alpha-\mathrm{Zr}$.

\section{Conclusions}

In this work, we have presented a comprehensive investigation correlating structural evolution, deformation mechanisms and mechanical strength of sputtered $\mathrm{Zr} / \mathrm{Nb}(\mathrm{hpc} / \mathrm{bcc}$ ) nano-multilayers with a periodicity $L$ in the range $6-167 \mathrm{~nm}$. XRD and electron diffraction analyses suggested a structural evolution in relation to $L$ especially for $\mathrm{Zr}$ layers with a consequent change in the crystallographic relationship between constituent layers. In particular, for $L \geq 27 \mathrm{~nm}$ the following relationship is found: $(0002)_{\mathrm{Zr}} / /(110)_{\mathrm{Nb}}$ (parallel to the substrate) with $<0001>_{\mathrm{Zr}} / /<110>_{\mathrm{Nb}}$ with inplane rotation of the crystals. On the other hand, for $L<27 \mathrm{~nm}$ the following relationship is found: $(10 \overline{1} 0)_{\mathrm{Zr}} / /(110)_{\mathrm{Nb}}$ (parallel to the substrate) with $<01 \overline{1} 1>_{\mathrm{Zr}} / /<0 \overline{1} \overline{1}>_{\mathrm{Nb}}$. High resolution STEM images also showed formation of specific interfaces for $L=6 \mathrm{~nm}$ characterised by different dislocation densities and resistance against dislocation transmission. 
The strength of $\mathrm{Zr} / \mathrm{Nb}$ NMMs in relation to $L$ was evaluated by nanoindentation. The Hall-Petch model described well the strengthening mechanism of $\mathrm{Zr} / \mathrm{Nb}$ NMMs for $L>60 \mathrm{~nm}$, while the refined CLS model came into picture for $27<L<60 \mathrm{~nm}$. A peak strength was reached for $L=27 \mathrm{~nm}$, while afterward a drop in hardness occurred for smaller $L$, which was not captured by the interface boundary strength (IBS) model (by taking into account only misfit and Koehler stresses). Structural analyses on compressed NMMs with $L \leq 27 \mathrm{~nm}$ revealed a change in the plastic behaviour of $\alpha$-Zr, which experienced a hard-to-soft transition. Such transition was attributed to the formation of weaker interfaces which extended the effective distance between strong barriers against dislocation transmission. Furthermore, the different crystallographic orientation found for $\alpha-\mathrm{Zr}$ for $L=6 \mathrm{~nm}$ favoured energetically less demanding slip systems. These structural features were introduced in the refined IBS model, which provided a more accurate quantification of the strength for $\mathrm{Zr} / \mathrm{Nb} \mathrm{NMMs}$ with small $L(<27 \mathrm{~nm})$.

\section{Acknowledgement}

Part of the work described has been supported by EPSRC under the program grant EP/K040375/1 'South of England Analytical Electron Microscope'. Part of the data published in this paper is available from the University of Southampton repository at http://dx.doi.org/10.5258/SOTON/401581.

\section{References}

[1] J.R. Greer, Jeff Th.M. De Hosson, Plasticity in small-sized metallic systems: Intrinsic versus extrinsic size effect, Prog. Mater. Sci. 56 (2011) 654-724.

[2] S.M. Spearing, Materials issues in microelectromechanical systems (MEMS), Acta Mater. 48 (2000) 179-196.

[3] S.N. Piramanayagam, Perpendicular recording media for hard disk drives, J. Appl. Phys. 102 (2007) 011301. 
[4] M. Andreas, T. Kentaro, T.M. David, A. Manfred, S. Yoshiaki, I. Yoshihiro, S. Shouheng, E.E. Fullerton, Magnetic recording: advancing into the future, J. Phys. D Appl. Phys. 35 (2002) R157.

[5] P. Chen, M. Zhu, Recent progress in hydrogen storage, Mater. Today 11 (2008) 36-43.

[6] N.Z. Abd.Khalim Khafidz, Z. Yaakob, K.L. Lim, S.N. Timmiati, The kinetics of lightweight solidstate hydrogen storage materials: A review, Int. J. Hydrogen Energy 41 (2016) 13131-13151.

[7] K. Holmberg, A. Matthews, H. Ronkainen, Coatings tribology - contact mechanisms and surface design, Tribol. Int. 31 (1998) 107-120.

[8] M. Callisti, M. Karlik, T. Polcar, Bubbles formation in helium ion irradiated Cu/W multilayer nanocomposites: Effects on structure and mechanical properties, J. Nucl. Mater. 473 (2016) 18-27. [9] M. Callisti, S. Lozano-Perez, T. Polcar, Structural and mechanical properties of $\gamma$-irradiated $\mathrm{Zr} / \mathrm{Nb}$ multilayer nanocomposites, Mater. Lett. 163 (2016) 138-141.

[10] M.J. Demkowicz, A. Misra, A. Caro, The role of interface structure in controlling high helium concentrations, Curr. Opin. Solid State Mater. Sci. 16 (2012) 101-108.

[11] I.J. Beyerlein, A. Caro, M.J. Demkowicz, N.A. Mara, A. Misra and B.P Uberuaga, Radiation damage tolerant nanomaterials, Mater. Today 16 (2013) 443-449.

[12] M.J. Demkowicz, R.G. Hoagland and J.P. Hirth, Interface Structure and Radiation Damage Resistance in Cu-Nb Multilayer Nanocomposites, Phys. Rev. Lett. 100, 136102 (2008).

[13] Q.M. Wei, N. Li, N. Mara, M. Nastasi, A. Misra, Suppression of irradiation hardening in nanoscale V/Ag multilayers, Acta Mater. 59 (2011) 6331-6340.

[14] E.G. Fu, A. Misra, H. Wang, L. Shao, X. Zhang, Interface enabled defects reduction in helium ion irradiated Cu/V nanolayers, J. Nucl. Mater. 407 (2010) 178-188.

[15] Y. Chen, Y. Liu, E.G. Fu, C. Sun, K.Y. Yu, M. Song, J. Li, Y.Q. Wang, H. Wang, X. Zhang, Unusual size-dependent strengthening mechanisms in helium ion-irradiated immiscible coherent Cu/Co nanolayers, Acta Mater. 84 (2015) 393-404. 
[16] A. Misra, J.P. Hirth, R.G. Hoagland, Length-scale-dependent deformation mechanisms in incoherent metallic multilayered composites, Acta Mater. 53 (2005) 4817-4824.

[17] J. Wang, A. Misra, An overview of interface-dominated deformation mechanisms in metallic multilayers, Curr. Opin. Solid State Mater. Sci. 15 (2011) 20-28.

[18] P.M. Anderson, C. Li, Hall-Petch relations for multi-layered materials, NanoStructured Materials 5(3) (1995) 349-362.

[19] R.E. Smallman, R.J. Bishop, Modern Physical Metallurgy and Materials Engineering, sixth ed., Linacre House, Jordan Hill, Oxford (UK), 1999.

[20] Y.Y. Lu, R. Kotoka, J.P. Ligda, B.B. Cao, S.N. Yarmolenko, B.E. Schuster, Q. Wei, The microstructure and mechanical behavior of $\mathrm{Mg} / \mathrm{Ti}$ multilayers as a function of individual layer thickness, Acta Mater. 63 (2014) 216-231.

[21] N. Li, K.Y. Yu, J. Lee, H. Wang, X. Zhang, Size dependent strengthening mechanisms in sputtered Fe/W multilayers, J. Appl. Phys. 107 (2010) 093503.

[22] J.Y. Zhang, Y. Liu, J. Chen, Y. Chen, G. Liu, X. Zhang, J. Sun, Mechanical properties of crystalline $\mathrm{Cu} / \mathrm{Zr}$ and crystal-amorphous $\mathrm{Cu} / \mathrm{Cu}-\mathrm{Zr}$ multilayers, Mater. Sci. Eng. A 552 (2012) 392398.

[23] Y. Liu, D. Bufford, H. Wang, C. Sun, X. Zhang, Mechanical properties of highly textured Cu/Ni multilayers, Acta Mater. 59 (2011) 1924-1933.

[24] Y. Chen, Y. Liu, C. Sun, K.Y. Yu, M. Song, H. Wang, X. Zhang, Microstructure and strengthening mechanisms in Cu/Fe multilayers, Acta Mater. 60 (2012) 6312-6321.

[25] E.G. Fu, N. Li, A. Misra, R.G. Hoagland, H. Wang, X. Zhang, Mechanical properties of sputtered $\mathrm{Cu} / \mathrm{V}$ and Al/Nb multilayer films, Mater. Sci. Eng A 493 (2008) 283-287.

[26] J. Wang, R.G. Hoagland, J.P. Hirth, A. Misra, Atomistic simulations of the shear strength and sliding mechanisms of copper - niobium interfaces, Acta Mater. 56 (2008) 3109-3119. 
[27] A. Misra, M. Verdier, Y.C. Lu, H. Kung, T.E. Mitchell, M. Nastasi, J.D. Embury, Structure and mechanical properties of $\mathrm{Cu}-\mathrm{X}(\mathrm{X}=\mathrm{Nb}, \mathrm{Cr}, \mathrm{Ni})$ nanolayered composites, Scripta Mater. 39 (1998) 555-560.

[28] S.I. Rao, P.M. Hazzledine, Atomistic simulations of dislocation-interface interactions in the CuNi multilayer system, Philos. Mag. A, 80 (2000), 2011-2040.

[29] J.S. Koehler, Attempt to Design a Strong Solid, Phys. Rev. B, 2 (1970), pp. 547-551.

[30] K.O. Schweitz, J. Chevallier, J. Bottiger, W. Matz, N. Schell, Hardness in Ag/Ni, Au/Ni and Cu/Ni multilayers, Philos. Mag. A, 81 (2001), pp. 2021-2032.

[31] K.Y. Yu, Y. Liu, S. Rios, H. Wang, X. Zhang, Strengthening mechanisms of Ag/Ni immiscible multilayers with fcc/fcc interface, Surf. Coat. Technol., 237 (2013), pp. 269-275.

[32] J. Li, Y. Chen, S. Xue, H. Wang, X. Zhang, Comparison of size dependent strengthening mechanisms in Ag/Fe and Ag/Ni multilayers, Acta Mater. 114 (2016) 154-163.

[33] R.G. Hoagland, T.E. Mitchell, J.P. Hirth, H. Kung, On the strengthening effects of interfaces in multilayer fee metallic composites, Philos. Mag. A, 82 (2002), pp. 643-664.

[34] Y. Liu, Y. Chen, K.Y. Yu, H. Wang, J. Chen, X. Zhang, Stacking fault and partial dislocation dominated strengthening mechanisms in highly textured Cu/Co multilayers, Int. J. Plast., 49 (2013), pp. 152-163.

[35] Y.T. Zhu, X.L. Wu, X.Z. Liaco, J. Narayan, L.J. Kecskes, S.N. Mathaudhu, Dislocation - twin interactions in nanocrystalline fcc metals, Acta Mater. 59 (2011) 812-821.

[36] J.J. Niu, J.Y. Zhang, G. Liu, P. Zhang, S.Y. Lei, G.J. Zhang, J. Sun, Size-dependent deformation mechanisms and strain-rate sensitivity in nanostructured $\mathrm{Cu} / \mathrm{X}(\mathrm{X}=\mathrm{Cr}, \mathrm{Zr})$ multilayer films, Acta Mater. 60 (2012) 3677-3689.

[37] M.A. Monclus, M. Karlik, M. Callisti, E. Frutos, J. LLorca, T. Polcar, J.M. Molina-Aldareguia, Microstructure and mechanical properties of physical vapor deposited $\mathrm{Cu} / \mathrm{W}$ nanoscale multilayers: Influence of layer thickness and temperature, Thin Solid Films 571 (2014) 275-282. 
[38] R. Raghavan, T.P. Harzer, V. Chawla, S. Djaziri, B. Phillipi, J. Wehrs, J.M. Wheeler, J. Michler, G. Dehm, Comparing small scale plasticity of copper-chromium nanolayered and alloyed thin films at elevated temperatures, Acta Mater. 93 (2015) 175-186.

[39] B.C. Kang, H.Y. Kim, O.Y. Kwon, S.H. Hong, Bilayer thickness effects on nanoindentation behavior of Ag/Ni multilayers, Scripta Mater. 57 (2007) 703-706.

[40] X.Y. Zhu, X.J. Liu, R.L. Zong, F. Zeng, F. Pan, Microstructure and mechanical properties of nanoscale Cu/Ni multilayers, Mater. Sci. Eng A 527 (2010) 1243-1248.

[41] K.Y. Yu, Y. Liu, S. Rios, H. Wang, X. Zhang, Strengthening mechanisms of Ag/Ni immiscible multilayers with fcc/fcc interface, Surf. Coat. Technol. 237 (2013) 269-275.

[42] A.F. Jankowski, J.P. Hayes, C.K. Saw, Dimensional attributes in enhanced hardness of nanocrystalline Ta-V nanolaminates, Philos. Mag., 87 (2007), pp. 2323-2334.

[43] B. Ham, X. Zhang, High strength Mg/Nb nanolayer composites, Mater. Sci. Eng A 528 (2011) 2028-2033.

[44] G.H. Yang, B. Zhao, Y. Gao, F. Pan, Investigation of nanoindentation on Co/Mo multilayers by the continuous stiffness measurement technique, Surf. Coat. Technol., 191 (2005), pp. 127-133.

[45] Q. Zhou, J.J. Li, F. Wang, P. Huang, K.W. Xu, T.J. Lu, Strain rate sensitivity of Cu/Ta multilayered films: Comparison between grain boundary and heterophase interface, Scripta Mater. 111 (2016) 123-126.

[46] J.Y. Zhang, J. Li, X.Q. Liang, G. Liu, J. Sun, Achieving optimum mechanical performance in metallic nanolayered Cu/X (X=Zr, Cr) micropillars, Scientific Report 4:4205, 1-9, 2014.

[47] J.Y. Zhang, X. Zhang, R.H. Wang, S.Y. Lei, P. Zhang, J.J. Niu, G. Liu, G.J. Zhang, J. Sun, Length-scale-dependent deformation and fracture behavior of $\mathrm{Cu} / \mathrm{X}(\mathrm{X}=\mathrm{Nb}, \mathrm{Zr})$ multilayers: The constraining effects of the ductile phase on the brittle phase, Acta Mater. 59 (2011) 7368-7379.

[48] J.Y. Zhang, S. Lei, Y. Liu, J.J. Niu, Y. Chen, G. Liu, X. Zhang, J. Sun, Length scale-dependent deformation behavior of nanolayered Cu/Zr micropillars, Acta Mater. 60 (2012) 1610-1622. 
[49] J.Y. Zhang, S. Lei, J. Niu, Y. Liu, G. Liu, X. Zhang, J. Sun, Intrinsic and extrinsic size effects on deformation in nanolayered $\mathrm{Cu} / \mathrm{Zr}$ micropillars: From bulk-like to small-volume materials behavior, Acta Mater. 60 (2012) 4054-4064.

[50] G.B. Thompson, R. Banerjee, S.A. Dregia, H.L. Fraser, Phase stability of bcc Zr in Nb/Zr thin film multilayers, Acta Mater. 51 (2003) 5285-5294.

[51] G.B. Thompson, R. Banerjee, H.L. Fraser, Predicting novel pseudomorphic phases in multilayers, Appl. Phys. Lett. 84 (2004) 1082-1084.

[52] R. Banerjee, P. Vasa, G.B. Thompson, H.L. Fraser, P. Ayyub, Proximity effect in Nb/Zr multilayers with variable Nb/Zr ratio, Solid State Commun. 127 (2003) 349-353.

[53] W.P. Lowe, T.H. Geballe, NbZr multilayers. I. Structure and superconductivity, Phys. Rev. Lett. 29 (1984) 4961-4966.

[54] A. Cavalleri, M. Dapor, F. Giacomozzi, L. Guzman, P.M. Ossi, M. Scotoni, Superconductivity in crystalline and amorphous Nb-Zr thin films, Mater. Sci. Eng. 99 (1988) 201-205.

[55] E. Tenckhoff. Deformation mechanisms, texture, and anisotropy in zirconium and zircaloy, ASTM special technical publication (STP 966), 1916 Race Street, Philadelphia, 1988.

[56] C.M. Eucken, A.M. Garde, Zirconium in the Nuclear Industry: Ninth International Symposium, ASTM (STP 1132), 1916 Race Street, Philadelphia, 1991.

[57] H.L. Heinisch, F. Gao, R.J. Kurtz, The effects of interfaces on radiation damage production in layered metal composites, J. Nucl. Mater. 329-333 (2004) 924-928.

[58] X.-M. Bai, A.F. Voter, R.G. Hoagland, M. Nastasi, B.P. Uberuaga, Efficient annealing of radiation damage near grain boundaries via interstitial emission, SCIENCE 327 (2010) 1631-1634.

[59] W.Z. Han, M.J. Demkowicz, E.G. Fu, Y.Q. Wang, A. Misra, Effect of grain boundary character on sink efficiency, Acta Mater. 60 (2012) 6341-6351.

[60] W.C. Oliver, G.M. Pharr, An improved technique for determining hardness and elastic modulus using load and displacement sensing indentation experiments, J. Mater. Res. 7 (1992) 1564. 
[61] A.C. Fischer-Cripps, Nanoindentation, second ed., Spring-Verlag, New York, 2004.

[62] B.M. Clemens, J.G. Gay, Effect of layer-thickness fluctuations on superlattice diffraction, Phys. Rev. B 35 (17) (1987) 9337-9340.

[63] Q. Yang, C. He, L.R. Zhao, J.-P. Immarigeon, Preferred orientation and hardness enhancement of TiN/CrN superlattice coatings deposited by reactive magnetron sputtering, Scripta Mater. 46 (2002) 293-297.

[64] W. Liu, A. Hu, S.-S. Jiang, Y. Qiu, W.H. Liu, Z.-Q. Wu, An x-ray diffraction study on Cu-Ti metallic multilayers, J. Phys.: Condens. Matter 1 (1989) 8771-8778.

[65] M. Birkholz, Thin Films Analyses by X-Ray Scattering, Wiley-VCH Verlag GmbH \& Co., KGaA, Weinheim, 2006.

[66] T.L. Wang, S.H. Liang, J.H. Li, K.P. Tai, B.X. Liu, Abnormal alloying behaviour observed in an immiscible Zr - Nb system, J. Phys. D: Appl. Phys. 41 (2008) 095310.

[67] J.Y. Zhang, Y.Q. Wang, G. Liu, J. Sun, Plastic deformation characteristics of Cu/X (X = Cu Zr, Zr) nanolayered materials, Appl. Surf. Sci. 321 (2014) 19-23.

[68] M.C. Liu, J.C. Huang, H.S. Chou, Y.H. Lai, C.J. Lee, T.G. Nieh, A nanoscaled underlayer confinement approach for achieving extraordinarily plastic amorphous thin film, Scripta Mater. 61 (2009) 840-843.

[69] X.Y. Zhu, J.T. Luo, F. Zeng, F. Pan, Microstructure and ultrahigh strength of nanoscale Cu/Nb multilayers, Thin Solid Films 520 (2011) 818-823.

[70] J. Dean, J. Campbell, G. Aldrich-Smith, T.W. Clyne, A critical assessment of the "stable indenter velocity” method for obtaining the creep stress exponent from indentation data, Acta Mater. 80 (2014) 56-66.

[71] M.Z. Wei, Z.H. Cao, J. Shi, G.J. Pan, L.J. Xu, X.K. Meng, Evolution of interfacial structures and creep behavior of Cu/Ta multilayers at room temperature, Mater. Sci. Eng. A 646 (2015) 163168. 
[72] X.Y. Zhu, X.J. Liu, R.L. Zong, F. Zeng, F. Pan, Microstructure and mechanical properties of nanoscale Cu/Ni multilayers, Mater. Sci. Eng. A 527 (2010) 1243-1248.

[73] J.Y. Zhang, Y.Q. Wang, K. Wu, P. Zhang, G. Liu, G.J. Zhang, J. Sun, Strain rate sensitivity of nanolayered $\mathrm{Cu} / \mathrm{X}(\mathrm{X}=\mathrm{Cr}, \mathrm{Zr})$ micropillars: Effects of heterophase interface/twin boundary, Mater. Sci. Eng. A 612 (2014) 28-40.

[74] L. Lu, T. Zhu, Y. Shen, M. Dao, K. Lu, S. Suresh, Stress relaxation and the structure sizedependence of plastic deformation in nanotwinned copper, Acta Mater. 57 (2009) 5165-5173.

[75] T. Zhu, J. Li, A. Samanta, A. Leach, K. Gall, Temperature and Strain-Rate Dependence of Surface Dislocation Nucleation, Phys. Rev. Lett. 100 (2008) 025502.

[76] P.M. Anderson, J.S. Carpenter, Estimates of interfacial properties in $\mathrm{Cu} / \mathrm{Ni}$ multilayer thin films using hardness data, Scripta Mater. 62 (2010) 325-328.

[77] Y.P. Li, J. Tan, G.P. Zhang, Interface instability within shear bands in nanoscale $\mathrm{Au} / \mathrm{Cu}$ multilayers, Scripta Mater. 59 (2008) 1226-1229.

[78] F. Wang, P. Huang, M. Xu, T.J. Lu, K.W. Xu, Shear banding deformation in Cu/Ta nanomultilayers, Mater. Sci. Eng. A 528 (2011) 7290-7294.

[79] D. Hull, D.J. Bacon, Introduction to Dislocations, Fifth ed. Elsevier Ltd, 2011.

[80] J.P. Escobedo, E.K. Cerreta, C.P. Trujillo, D.T. Martinez, R.A. Lebensohn, V.A. Webster, G.T. Gray, Influence of texture and test velocity on the dynamic, high-strain, tensile behavior of zirconium, Acta Mater. 60 (2012) 4379-4392.

[81] J.F. Bingert, T.A. Mason, G.C. Kaschner, P.J. Maudlin, G.T. Gray, Deformation twinning in polycrystalline Zr: Insights from electron backscattered diffraction characterization, Metall. Mater. Trans. 33 A (2002) 955-963.

[82] L.B. Addessio, E.K. Cerreta, G.T. Gray, Mechanical behavior of zirconium and hafnium in tension and compression, Metall. Mater. Trans. 36 A (2005) 2893-2903. 
[83] G. Proust, C.N. Tome, G.C. Kaschner, Modeling texture, twinning and hardening evolution during deformation of hexagonal materials, Acta Mater. 55 (2007) 2137-2148.

[84] J. Gong, T.B. Britton, M.A. Cuddihy, F.P.E. Dunne, A.J. Wilkinson, <a> Prismatic, <a> basal, and $<\mathrm{c}+\mathrm{a}>$ slip strengths of commercially pure Zr by micro-cantilever tests, Acta Mater. 96 (2015) 249-257. 
Figures

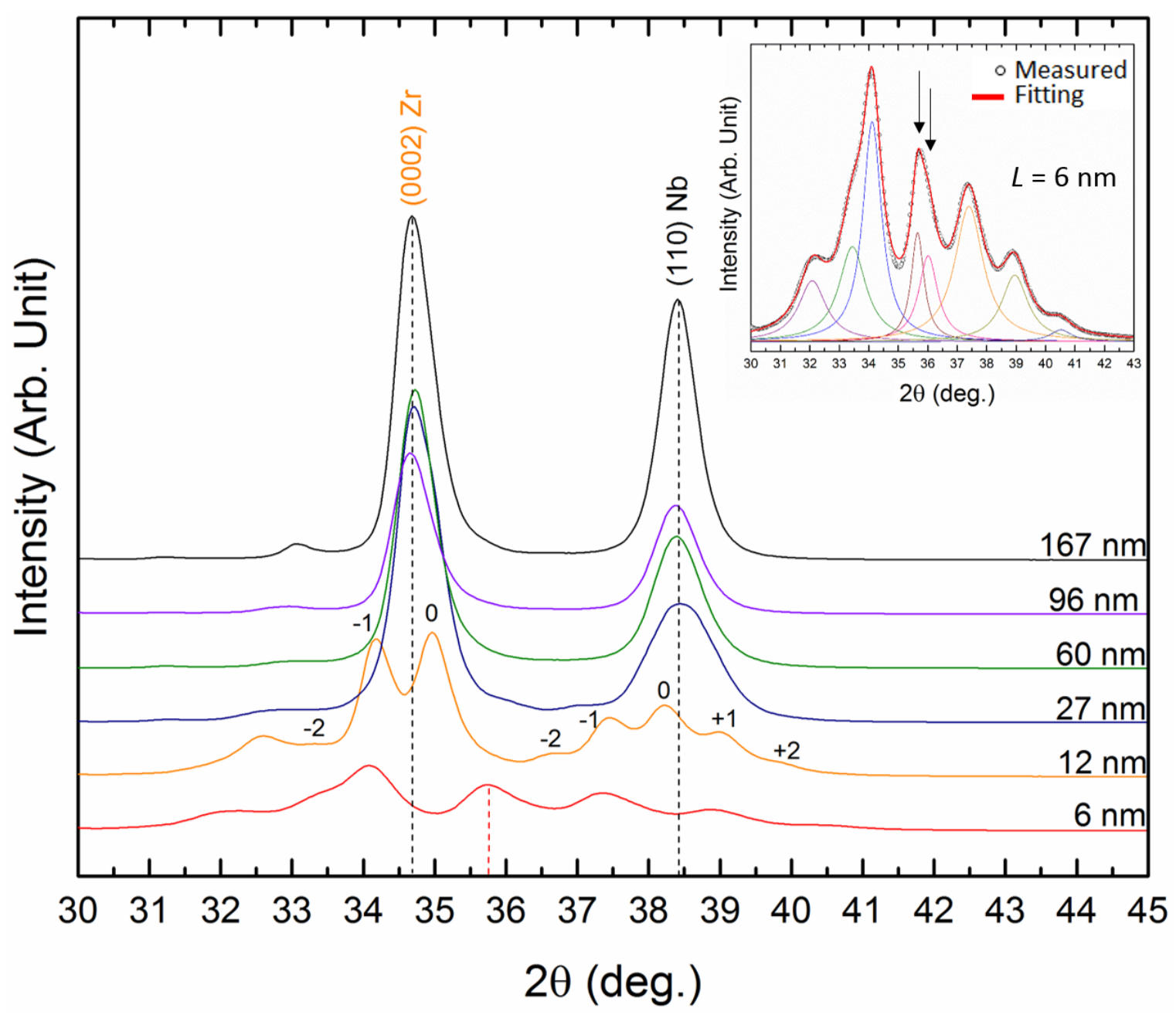

Fig. 1. XRD patterns of the as-deposited $\mathrm{Zr} / \mathrm{Nb}$ NMMs with different periodicities $(L)$. The inset shows the deconvolution of the XRD profile for $L=6 \mathrm{~nm}$. 

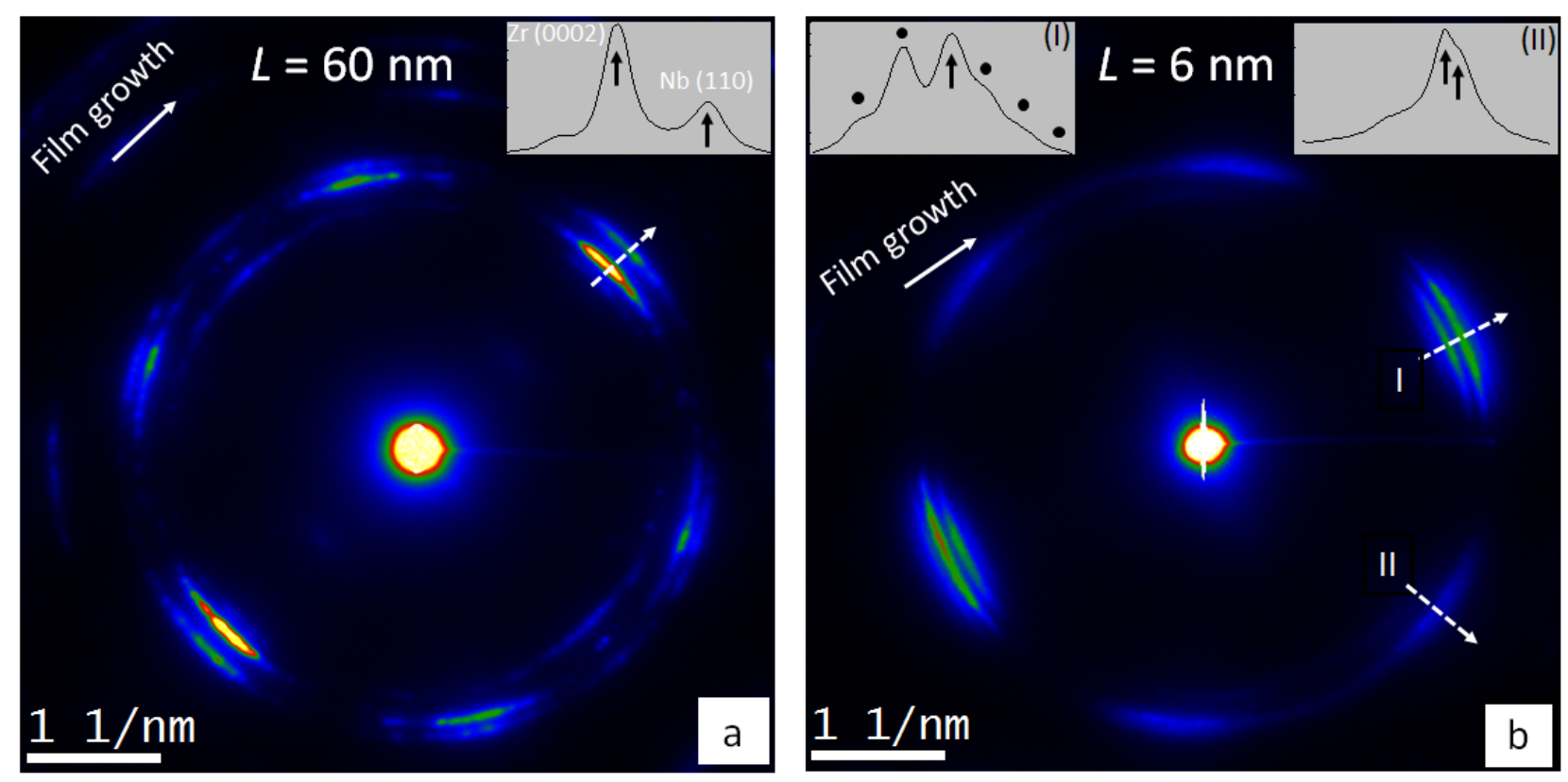

Fig. 2. Selected area electron diffraction (SAD) patterns acquired on the cross-section of $\mathrm{Zr} / \mathrm{Nb}$ NMMs with (a) $L=60 \mathrm{~nm}$ and (b) $L=6 \mathrm{~nm}$. The insets in each figure show the line profile across diffraction rings. 


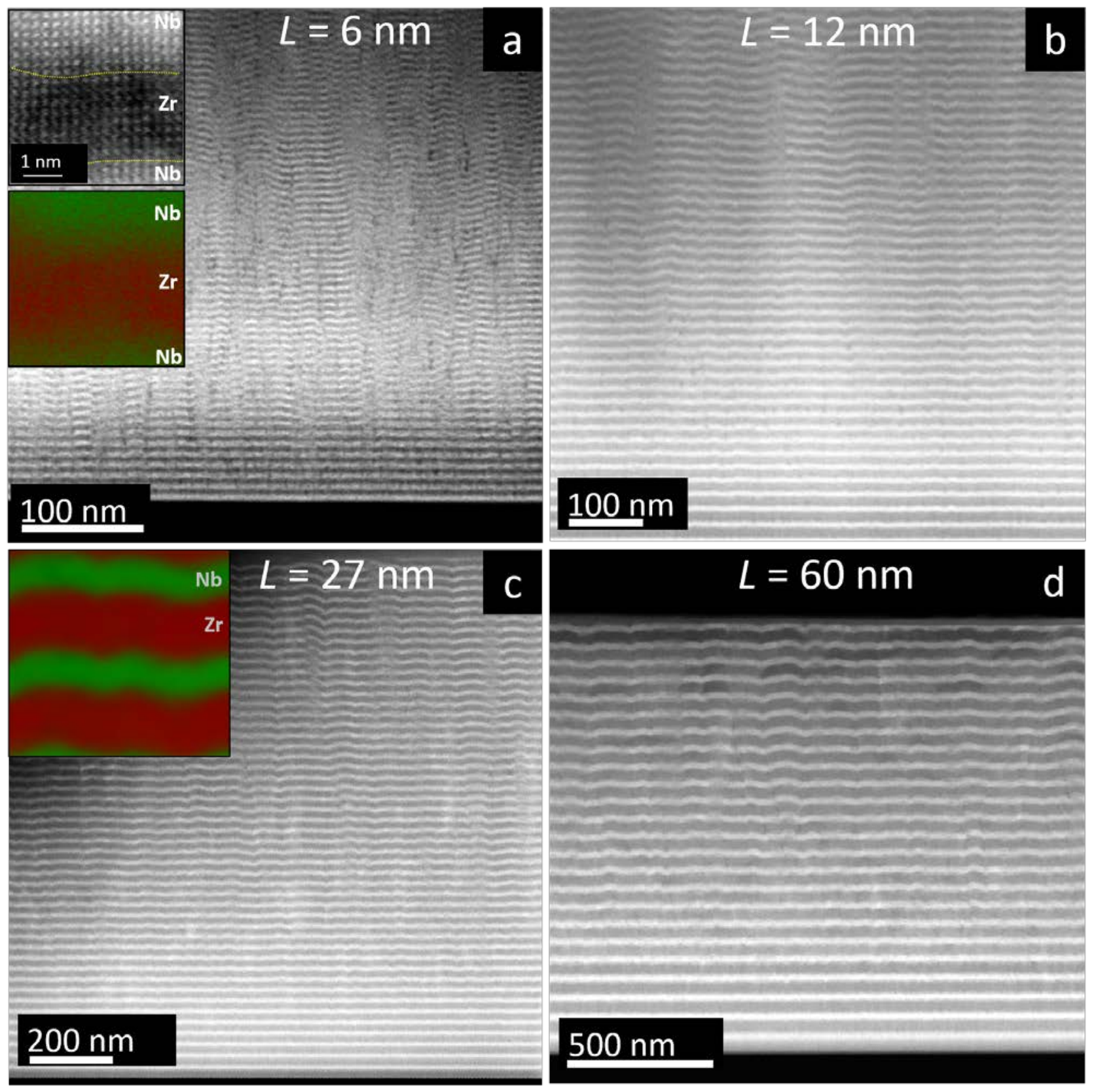

Fig. 3. Cross-sectional HAADF-STEM images of the as-deposited NMMs with different periodicities L: (a) 6, (b) 12, (c) 27 and (d) $60 \mathrm{~nm}$. Insets in (a) and (c) show the EELS phase maps for Zr and Nb. 

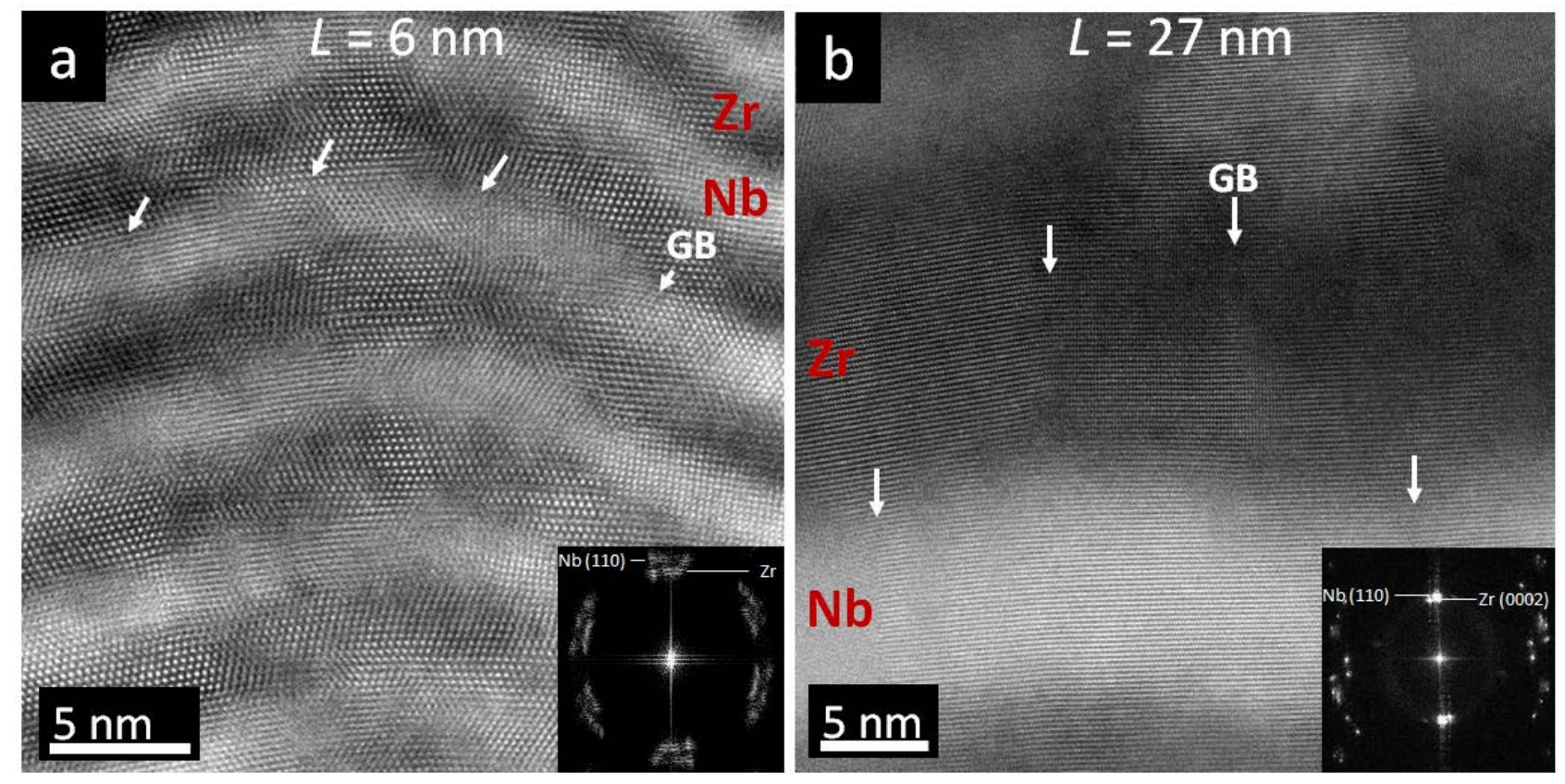

Fig. 4. High resolution HAADF-STEM images for a periodicity $L$ of (a) 6 and (b) $27 \mathrm{~nm}$. Arrows highlight grain boundaries (GBs) within the layers. Unindexed spots in the inset of Fig. 4b are caused by the slightly tilted structure exhibited by some grains in the Zr layer. 

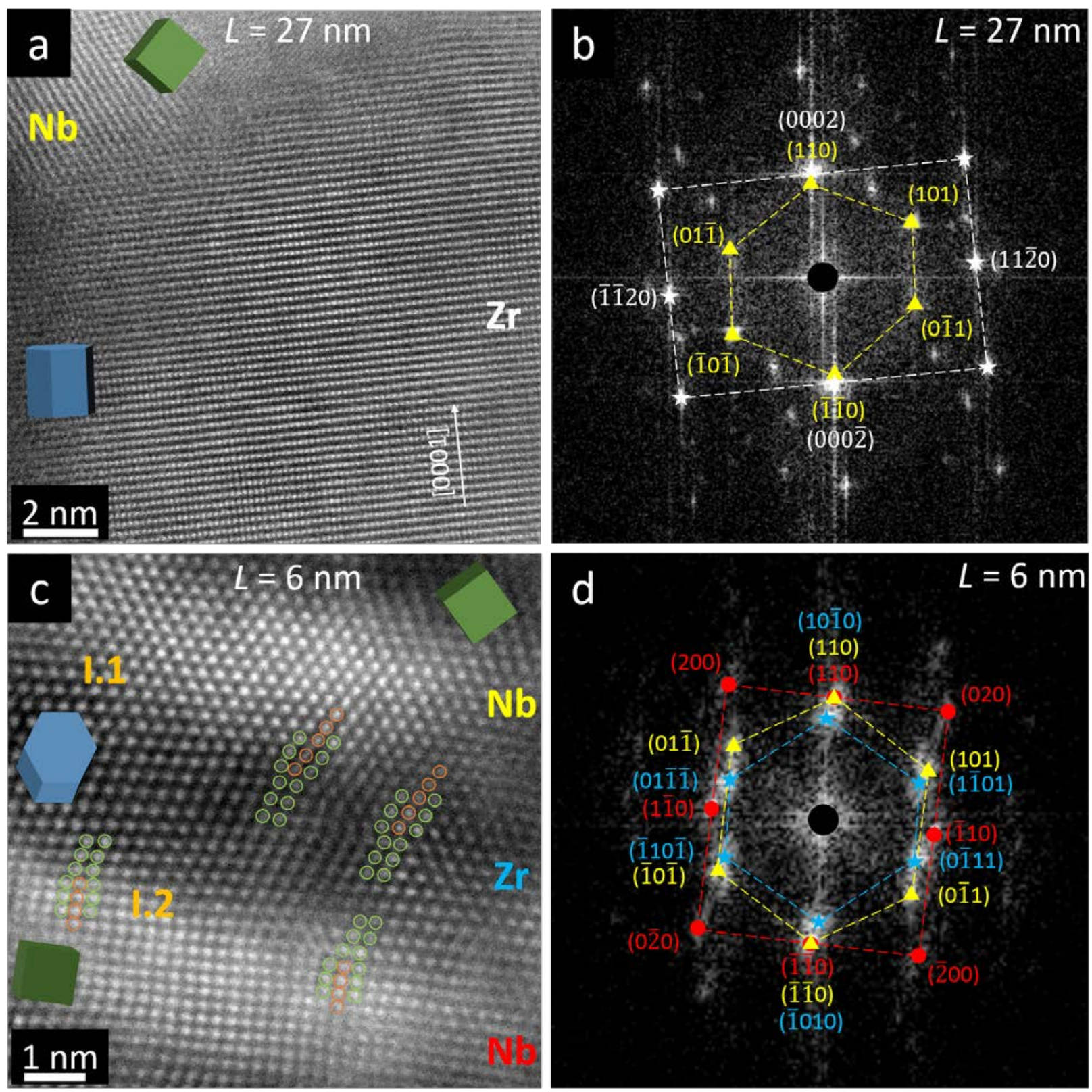

Fig. 5. High resolution HAADF-STEM images and corresponding FFT for $\mathrm{Zr} / \mathrm{Nb}$ NMMs with a periodicity $L$ of (a) - (b) $27 \mathrm{~nm}$ and (c) - (d) $6 \mathrm{~nm}$. Cartoons for cubic and hexagonal close-packed structures are used to indicate roughly the crystallographic orientation of the constituent elements for different periodicities. In (c) dislocations are highlighted by circles, while $I .1$ and $I .2$ indicate interfaces of different nature and interfacial dislocation densities. 

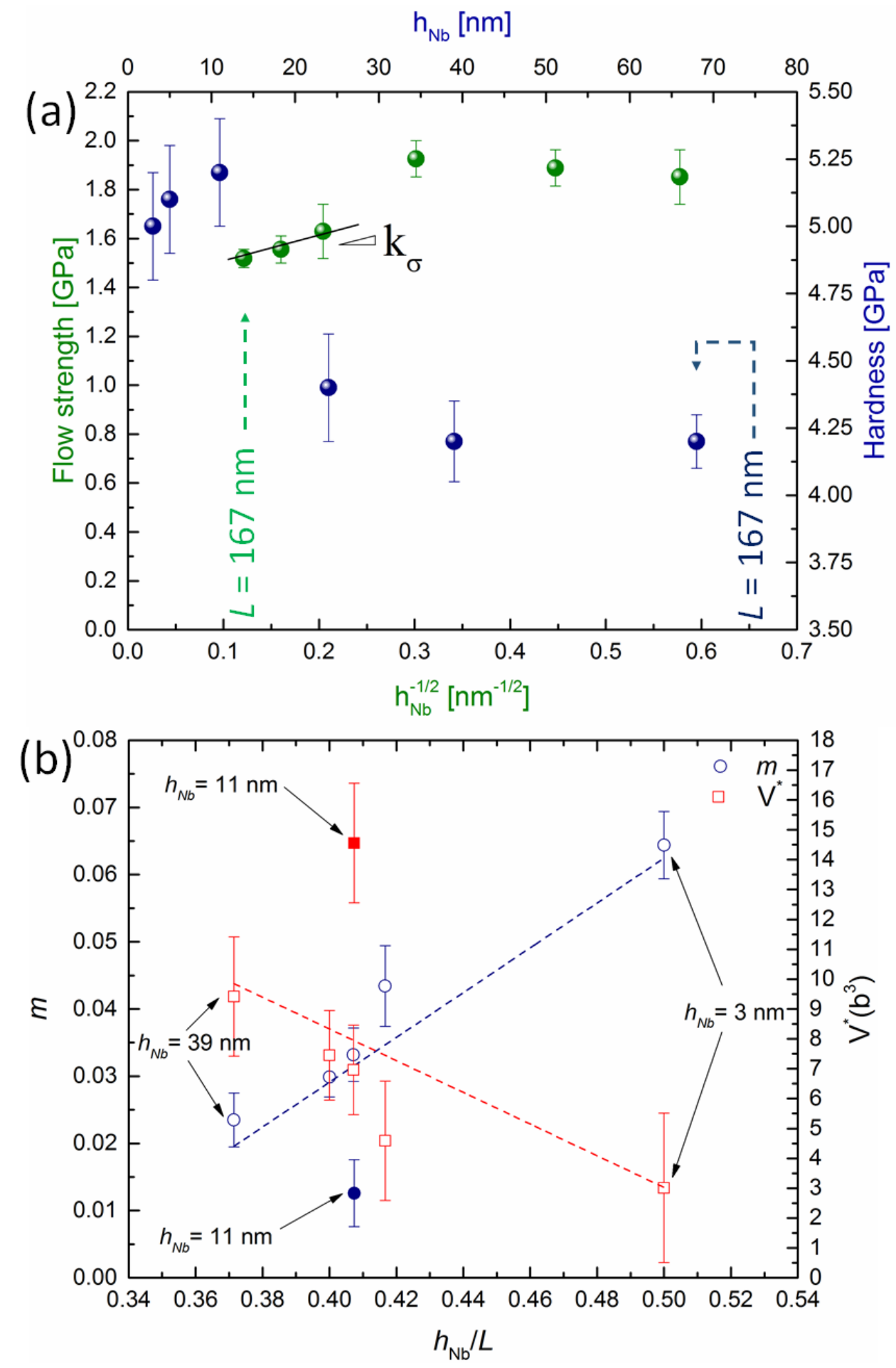

Fig. 6. Mechanical properties measured by nanoindentation: (a) flow strength and hardness vs intrinsic length scales, (b) strain rate sensitivity $(m)$ and activation volume $\left(V^{*}\right)$ vs the relative amount of $\mathrm{Nb}$; dashed lines in (b) are shown as guidelines. 


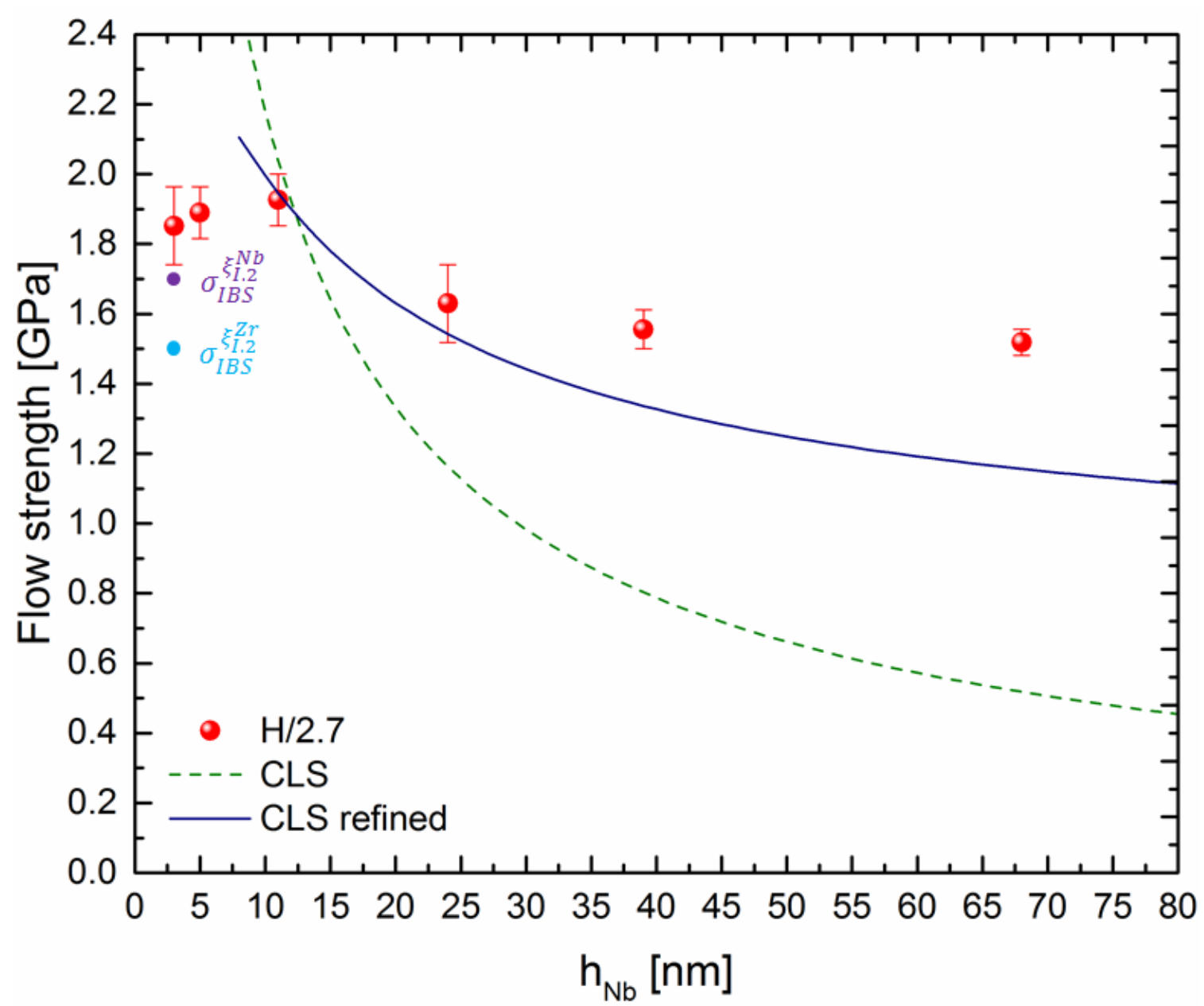

Fig. 7. Flow strength of the $\mathrm{Zr} / \mathrm{Nb}$ NMMs (estimated as the nanoindentation hardness divided by a Taylor factor of 2.7) as a function of $h_{N b}$. Calculation from different models are also depicted for comparison. $\sigma_{I B S}^{\xi_{I .2}^{Z r}}$ and $\sigma_{I B S}^{\xi_{I .2}^{N b}}$ are the IBS stresses calculated by using the misfit strains in $\mathrm{Zr}$ and $\mathrm{Nb}$ lattices, respectively. See text for details. 


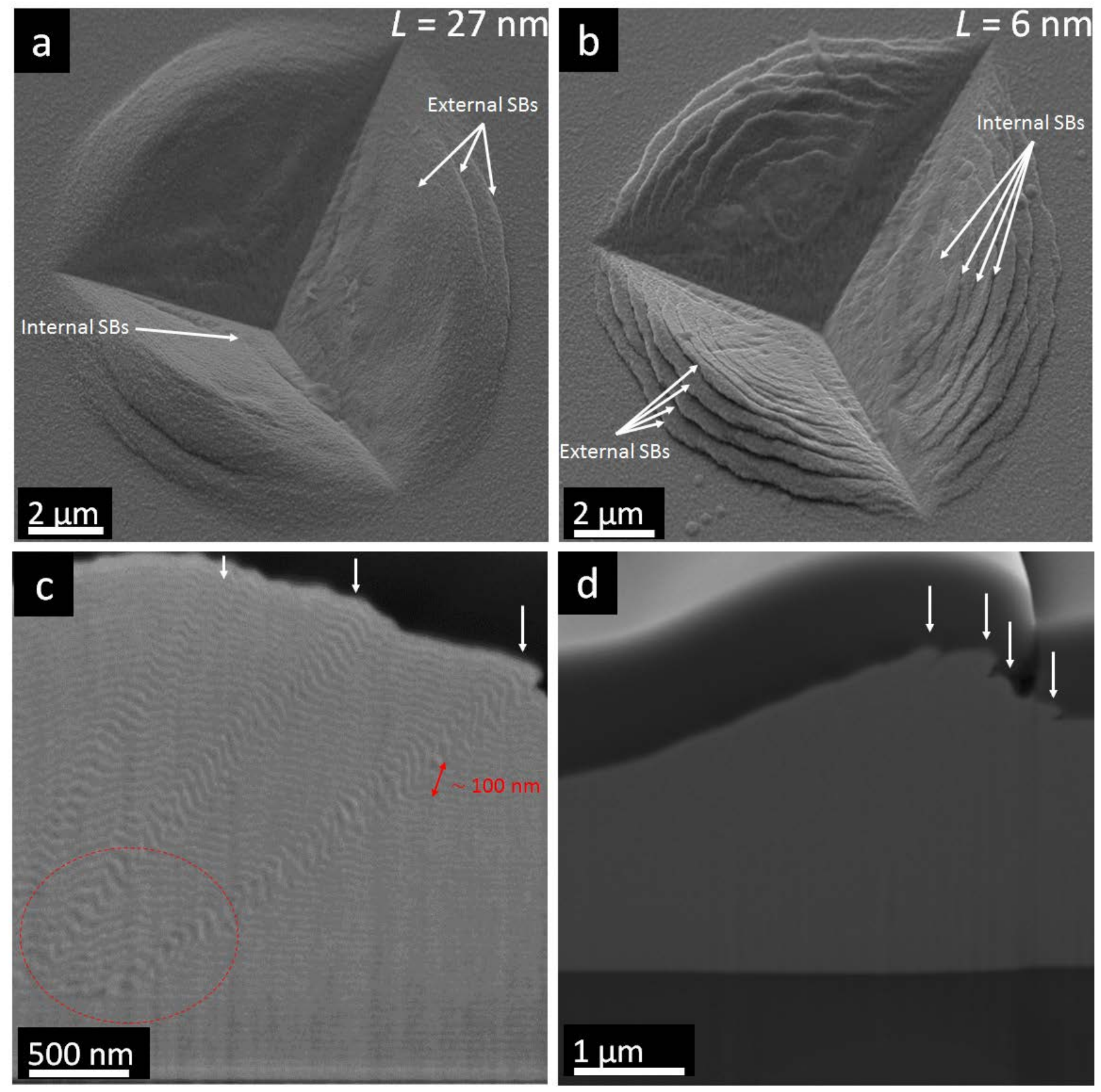

Fig. 8. SEM images of plastically deformed $\mathrm{Zr} / \mathrm{Nb}$ NMMs by means of nanoindentation. Internal and external shear bands (SBs) are highlighted by arrows on top view and cross-section images for NMMs with a periodicity $L$ of $27 \mathrm{~nm}$ in (a) - (c) and $6 \mathrm{~nm}$ in (b) - (d). 

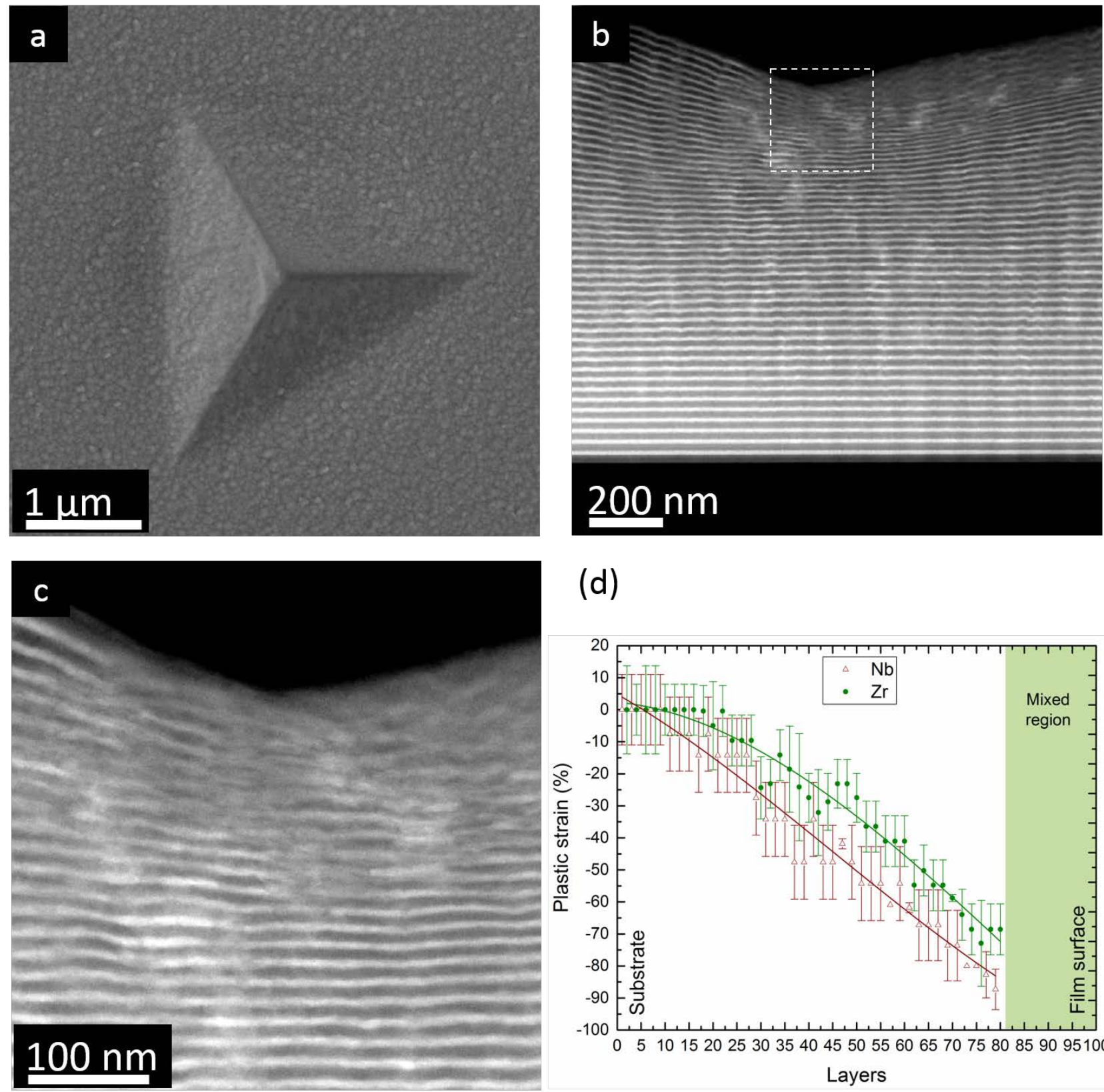

(d)

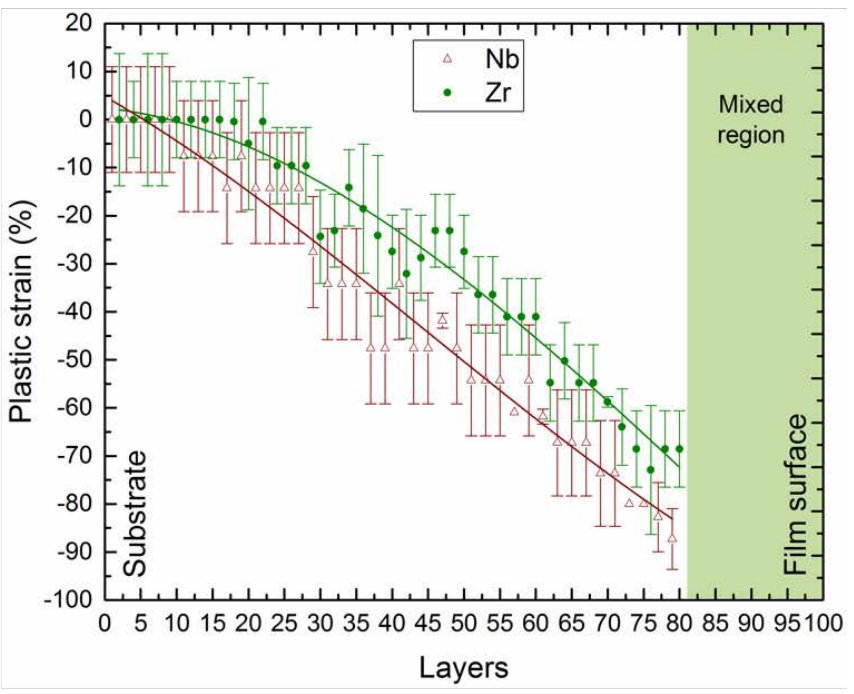

Fig. 9. Plastically deformed $\mathrm{Zr} / \mathrm{Nb}$ NMMs with $L=27 \mathrm{~nm}$ : (a) top view SEM image of the indent produced with a load of $50 \mathrm{mN}$; (b) cross-sectional HAADF-STEM image of the indent in (a); (c) detail of the layered structure underneath the indented surface highlighted in (b); (d) plastic strain $\varepsilon_{p}$ for each constituent element along the loading axis as a function of the number of layers across the film thickness. Fitting curves are reported to facilitate the interpretation of numerical data. 

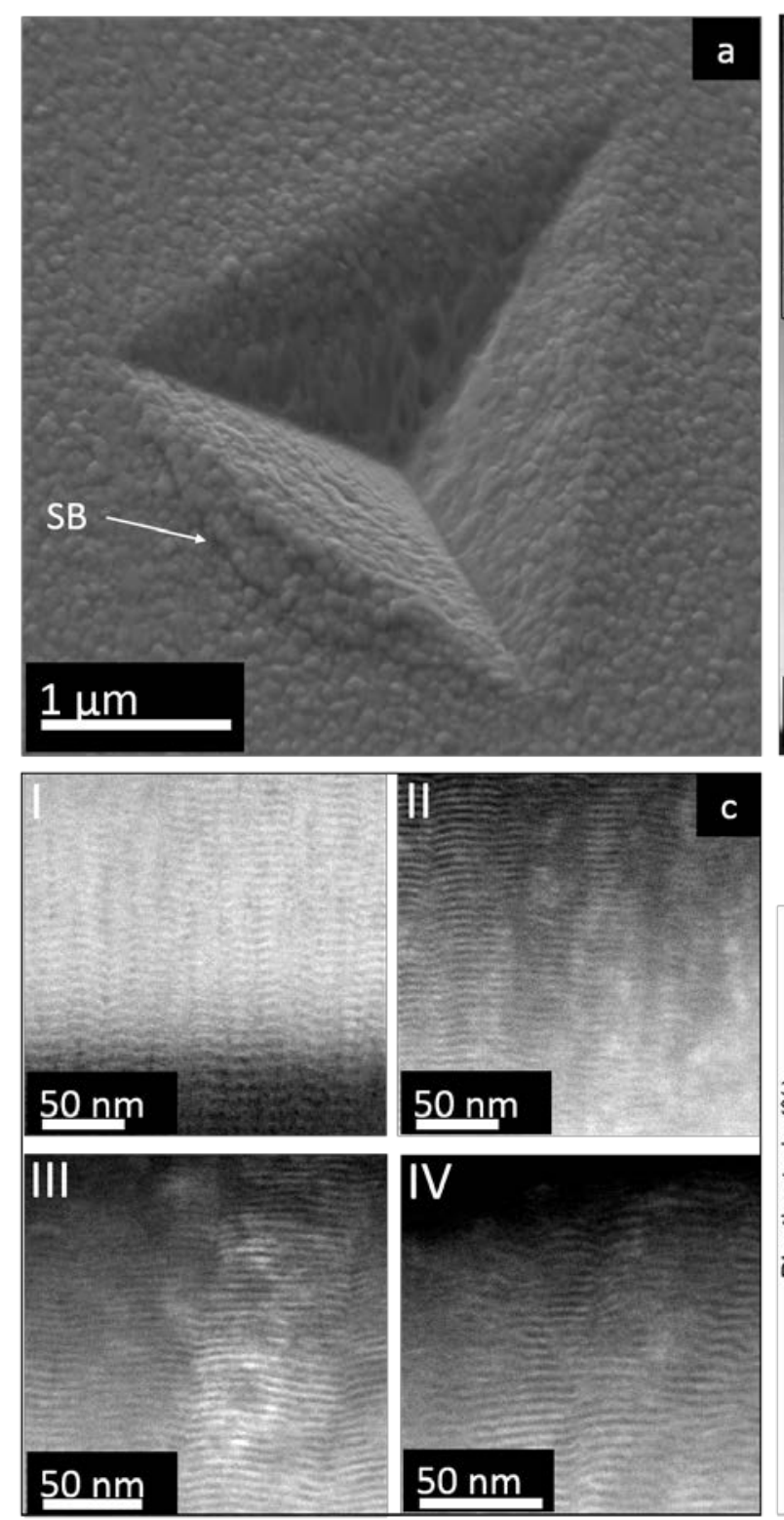

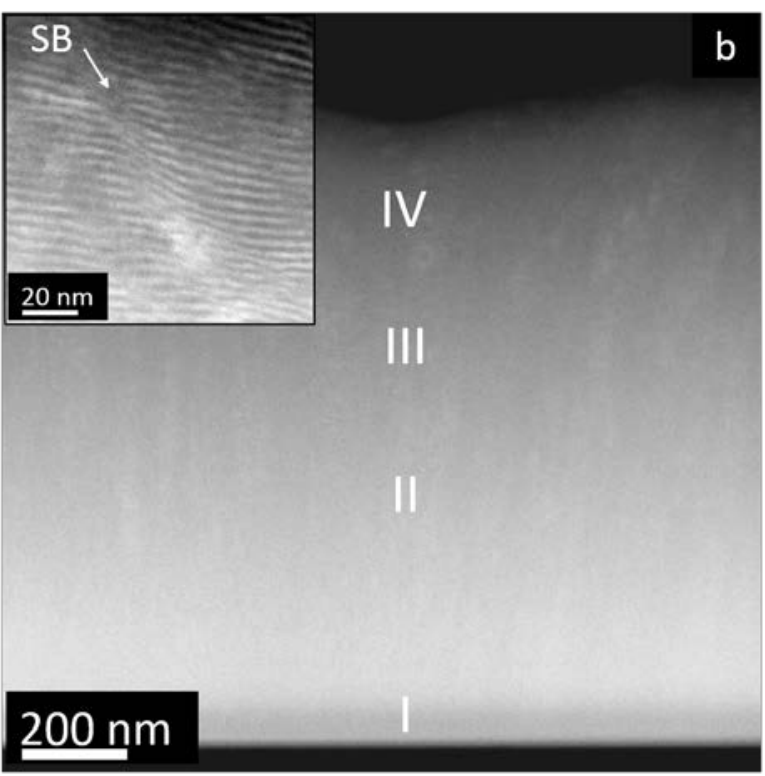

(d)

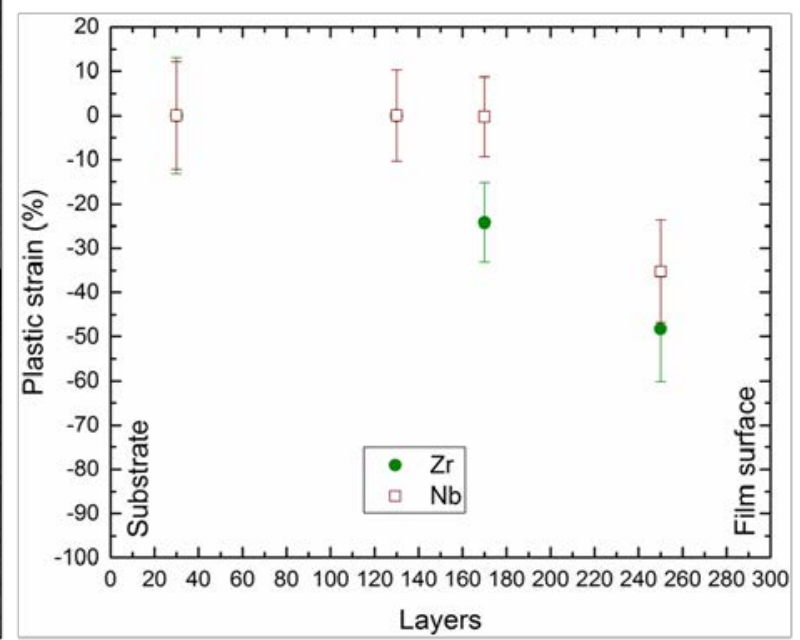

Fig. 10. Plastically deformed $\mathrm{Zr} / \mathrm{Nb}$ NMMs with $L=6 \mathrm{~nm}$ : (a) top view SEM image of the indent produced with a load of $50 \mathrm{mN}$. (b) Cross-sectional HAADF-STEM image of the indent in (a); the inset shows the cross-section view of the shear band (SB) highlighted in (a). (c) Details of the layered structure in various regions indicated in (b) underneath the indented surface. (d) Plastic strain $\varepsilon_{p}$ for each constituent element along the loading axis as a function of the number of layers across the film thickness. 


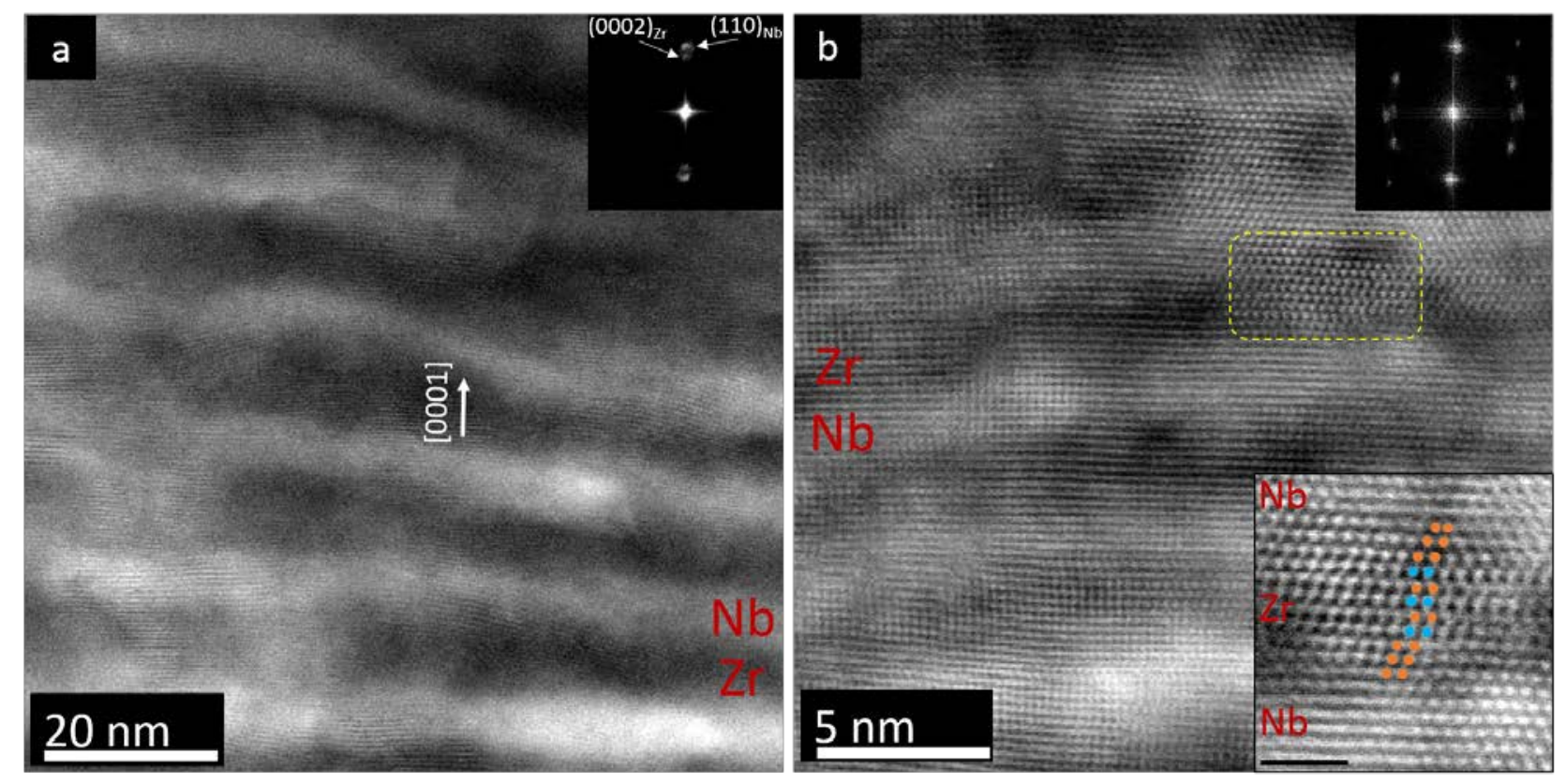

Fig. 11. High resolution HAADF-STEM images of the layered structure along the loading axis after compression tests for a periodicity $L$ of (a) 27 where the $(0002)_{\mathrm{Zr}} / /(100)_{\mathrm{Nb}}$ orientation relationship is retained (see inset). In (b) a twinned structure formed after compression test in $\mathrm{Zr} / \mathrm{Nb}$ NMM with $L$ $=6 \mathrm{~nm}$ is highlighted and a detail is shown in the inset (scale bar $=1 \mathrm{~nm})$. 


\section{Tables}

Tab. 1. Structural properties of the as-deposited $\mathrm{Zr} / \mathrm{Nb}$ NMMs determined by XRD. See text for details. Values indicated with “*” are not attributed to the (0002)Zr plane. See text for details. Layers thickness was measured on STEM (T) and SEM (S) micrographs.

\begin{tabular}{lcccccccc}
\hline Film & $\begin{array}{c}\text { Crystallite size } \\
\text { (XRD) [nm] }\end{array}$ & \multicolumn{2}{c}{$\begin{array}{c}\text {-spacing } \\
{[\mathrm{nm}]}\end{array}$} & $\begin{array}{c}\text { Layer } \\
\text { thickness } \\
{[\mathrm{nm}]}\end{array}$ & $\begin{array}{c}\text { Periodicity } \\
(\mathrm{L})[\mathrm{nm}]\end{array}$ & $\begin{array}{c}\text { Film thickness } \\
{[\mathrm{nm}]}\end{array}$ \\
\hline $\mathrm{Zr} / \mathrm{Nb}_{6}$ & 11 & 5 & $0.2516^{*}$ & 0.2492 & $3(\mathrm{~T})$ & $3(\mathrm{~T})$ & 6 & 1480 \\
$\mathrm{Zr} / \mathrm{Nb}_{12}$ & 13 & 7 & 0.2560 & 0.2353 & $7(\mathrm{~T})$ & $5(\mathrm{~T})$ & 12 & 1500 \\
$\mathrm{Zr} / \mathrm{Nb}_{27}$ & 14 & 9 & 0.2584 & 0.2339 & $16(\mathrm{~T})$ & $11(\mathrm{~T})$ & 27 & 1350 \\
$\mathrm{Zr} / \mathrm{Nb}_{60}$ & 16 & 12 & 0.2581 & 0.2342 & $36(\mathrm{~T})$ & $24(\mathrm{~T})$ & 60 & 1500 \\
$\mathrm{Zr} / \mathrm{Nb}_{96}$ & 17 & 14 & 0.2583 & 0.2343 & $57(\mathrm{~S})$ & $39(\mathrm{~S})$ & 96 & 1350 \\
$\mathrm{Zr} / \mathrm{Nb}_{167}$ & 18 & 18 & 0.2583 & 0.2341 & $99(\mathrm{~S})$ & $68(\mathrm{~S})$ & 167 & 1350 \\
\hline
\end{tabular}

Tab. 2. Mechanical properties of the as-deposited $\mathrm{Zr} / \mathrm{Nb}$ NMMs measured by nanoindentation. The elastic modulus $(E)$ of the films was calculated from the reduced elastic modulus by using a Poisson's ratio and elastic modulus for the diamond tip of 0.07 and $1141 \mathrm{GPa}$, respectively. An average Poisson's ratio for the NMMs was calculated by using $v_{\mathrm{Zr}}=0.34$ and $v_{\mathrm{Nb}}=0.4$.

\begin{tabular}{ccccc}
\hline Film & H (GPa) & E (GPa) & $\mathbf{V}^{*}\left(\mathbf{b}^{\mathbf{3}}\right)$ & $\boldsymbol{m}$ \\
\hline $\mathrm{Zr} / \mathrm{Nb}_{6}$ & $5 \pm 0.3$ & $119.35 \pm 5$ & 3 & 0.06 \\
$\mathrm{Zr} / \mathrm{Nb}_{12}$ & $5.1 \pm 0.2$ & $124.58 \pm 3$ & 4.6 & 0.04 \\
$\mathrm{Zr} / \mathrm{Nb}_{27}$ & $5.2 \pm 0.2$ & $136.52 \pm 5$ & 14.6 & 0.01 \\
$\mathrm{Zr} / \mathrm{Nb}_{60}$ & $4.4 \pm 0.3$ & $124.91 \pm 3$ & 7.5 & 0.03 \\
$\mathrm{Zr} / \mathrm{Nb}_{96}$ & $4.2 \pm 0.1$ & $121.74 \pm 3$ & 9.4 & 0.02 \\
$\mathrm{Zr} / \mathrm{Nb}_{167}$ & $4.2 \pm 0.1$ & $128.97 \pm 4$ & 7 & 0.03 \\
\hline
\end{tabular}




\section{Graphical abstract}
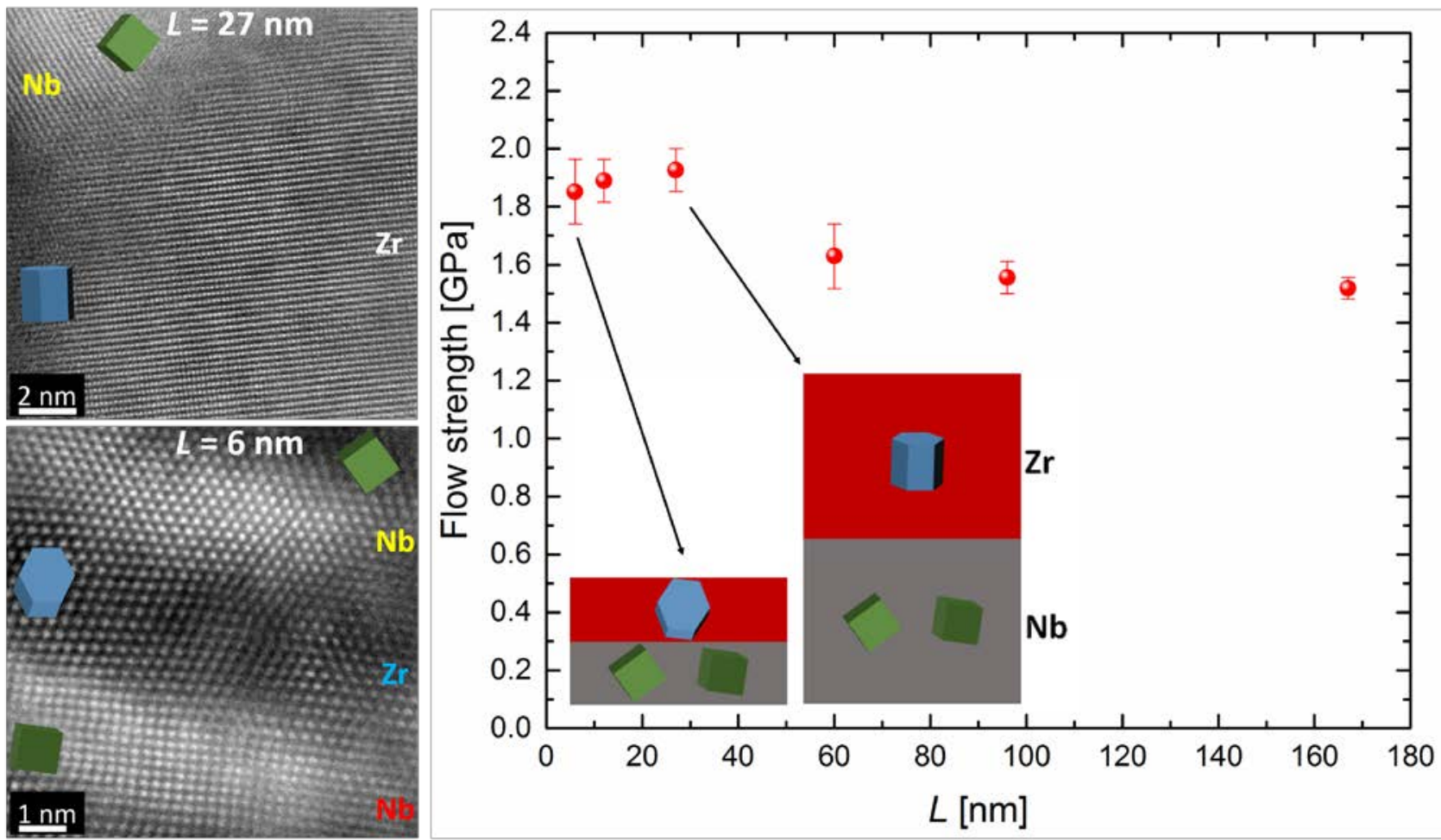